\title{
INTRODUCTION TO MESOSCOPIC ELECTRON TRANSPORT
}

\author{
LEO P. KOUWENHOVEN \\ Dept. of Applied Physics and DIMES \\ Delft University of Technology, 2600 GA Delft, The Netherlands \\ GERD SCHÖN \\ Institut für Theoretische Festkörperphysik \\ Universtität Karlsruhe, 76128 Karlsruhe, Germany
}

AND

LYDIA L. SOHN

Dept. of Physics, Princeton University

Princeton, NJ 08544-0708, USA

In this introductory chapter several basic concepts Trelevant for mesoscopic electron transport will be described. The aim is to provide a basis for several of the following Chapters of this volume. WeTthereforeГdescribe in the first Section various aspects of electron quantum transport in twodimensional electron gases. This includes an estimate of typical material parameters as well as comments on the fabrication. We then describe the quantization of the conductance in point contacts and the edge state picture of the Quantum Hall effect. In the second Section we describe the theory of single-electron tunneling in systems with strong charging and Coulomb-blockade effects. Here we restrict ourselves to the simplest case where low order perturbation theory is sufficient. We first discuss metallic low-capacitance junction systems and then indicate the relevant extensions when dealing with transport through quantum dots with discrete levels. Many further Tequally important aspects of mesoscopic transport can not be covered here. Examples are interference and weak localization effects $\Gamma$ level statistics or the many body description of solids. FortunatelyГsome of those will be covered in the more specialized Chapter of this volume. 
(a)

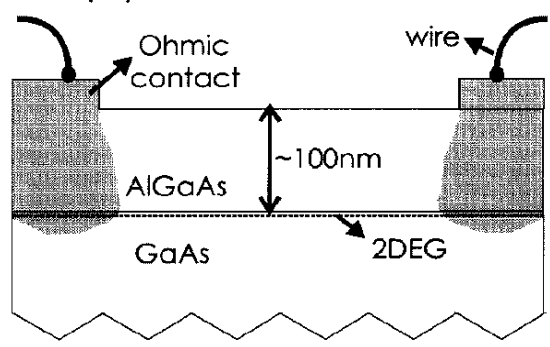

(b)

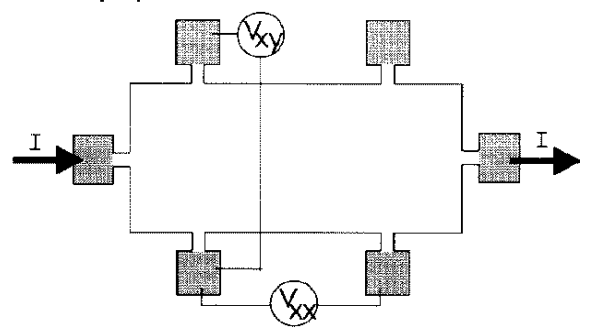

Figure 1. (a) Schematic cross section of a GaAs/AlGaAs heterostructure. The 2DEG is located at the interface between the GaAs substrate and the AlGaAs top layer. (b) Hall bar with six Ohmic contacts (shaded squares).

\section{Electron Transport}

\subsection{DEG SYSTEMS AND THE QUANTUM HALL EFFECT}

In this Section we introduce basic properties of a two-dimensional electron gas (2DEG) in a GaAs/AlGaAs heterostructure (see Fig. 1a). On the GaAs substrate a layer of typically $100 \mathrm{~nm}$ AlGaAs is grown. Somewhere halfway in the $\mathrm{AlGaAs}$ layer there is a thin layer where the Ga atoms are replaced by $\mathrm{Si}$ donor atoms. With a proper amount of $\mathrm{Si}$ one finds that at low temperature the only mobile electrons are located at the GaAs/AlGaAs interface. These free electrons are attracted by the GaAs since they can lower their energy in this smaller band gap material. They are also held as close as possible to their ionized $\mathrm{Si}^{+}$donors and thus they form a thin conducting layer near the GaAs/AlGaAs interface (for a review on growth of $\mathrm{GaAs}$ heterostructures see Ref. [1]). Since GaAs and AlGaAs can form a nearly perfect interface on the atomic scale and since the Si donors are spatially separated $\Gamma$ the electrons experience very little scattering. Typical mean free paths are $10 \mu \mathrm{m}$ and the record is close to $100 \mu \mathrm{m}$. Before we discuss ballistic mesoscopic devices we first review a few important electron transport properties of 2DEG's. In Table 1.1 we have summarized a number of useful relations and some typical values for GaAs/AlGaAs heterostructures.

For transport experiments one first defines a so-called Hall-bar of typically $0.1 \mathrm{~mm} \times 1 \mathrm{~mm}$. At the edge of the Hall-bar one then locally evaporates a number of metal squares of roughly $50 \mu \mathrm{m} \times 50 \mu \mathrm{m}$ in size. By heating the whole sample this metal diffuses into the semiconductor where at some point it makes electrical contact to the 2DEG. Good contacts are characterized by linear current-voltage traces and are therefore called Ohmic contacts. Fig. 1b shows a Hall-bar with 6 Ohmic contacts in a configuration that allows measurements of the Hall resistance $R_{\mathrm{xy}}$ and the longitudinal 


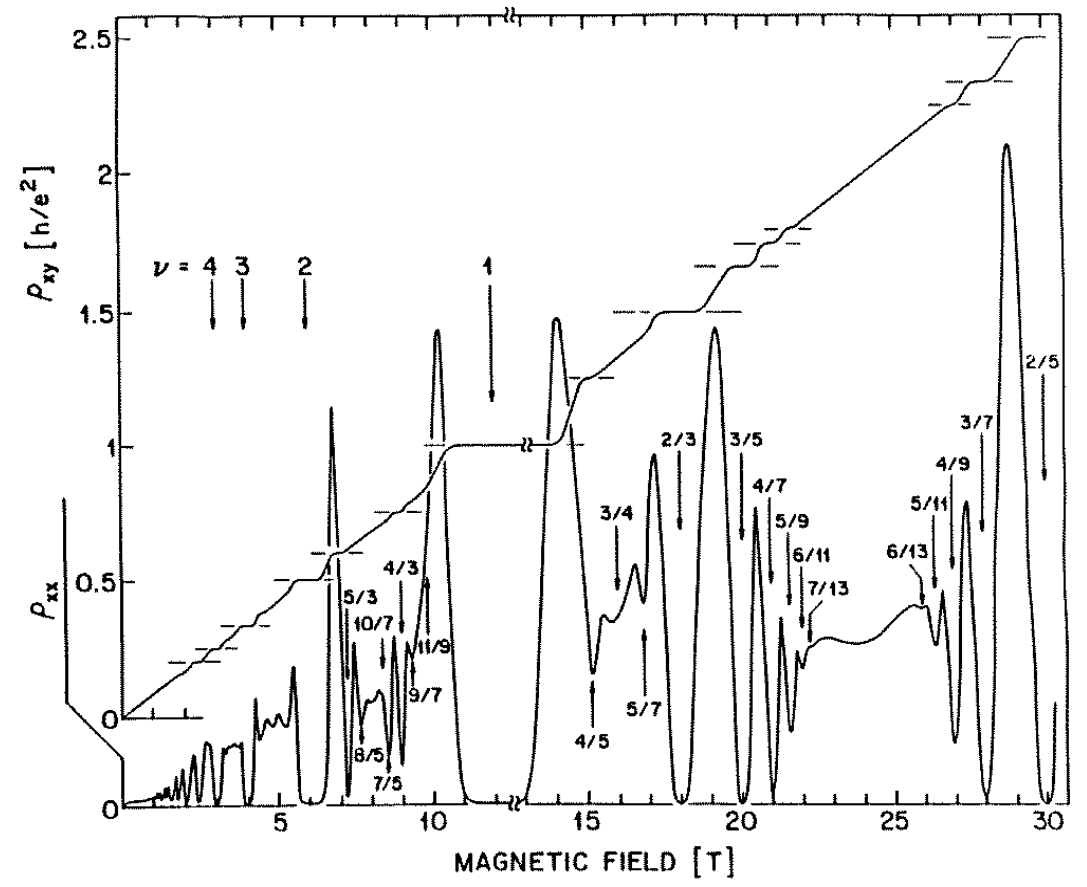

Figure 2. Measurement of the Hall resistance $R_{\mathrm{xy}}$ and the longitudinal resistance $R_{\mathrm{xx}}$ at $0.1 \mathrm{~K}$. The mobility is $\mu=1.5 \cdot 10^{6} \mathrm{~cm}^{2} / \mathrm{Vs}$ the temperature is $T=80 \mathrm{mK}$. (Figure provided by R. L. Willett.)

resistance $R_{\mathrm{xx}}$. The sample is current biased and resulting voltages can be measured as a function of magnetic field.

Fig. 2 shows a remarkable measurement of $R_{\mathrm{xy}}$ and $R_{\mathrm{xx}}$ as a function of magnetic field measured at a temperature of $80 \mathrm{mK}$. The Hall resistance shows plateaus where the longitudinal resistance has minima of virtually zero resistance. The striking aspect of this data is that the plateaus are precisely quantized at integer and fractional multiples of $h / e^{2}$. The integer plateaus are known as the integer quantum Hall effect (QHE) Tand correspondingly the fractional plateaus are referred to as the fractional QHE. The precision of the quantization of the former is so accurate that it now forms the international standard of resistance. An important quantity in the quantum Hall regime is the filling factor $\nu=h n_{s} / e B$. It is equal to the number of electrons divided by the number of flux quanta $\Phi_{0}=h / e$. For filling factor $\nu=1$ the system is in the center of the first platean at $h / e^{2} \Gamma$ for $\nu=2$ in the center of the second plateau at $h / 2 e^{2} \Gamma$ and so forth. The filling factor is $\Gamma$ for instance $\Gamma$ convenient for determining the electron density. The oscillations in the longitudinal resistance $\Gamma$ which have minima 
at the same magnetic fields where the Hall resistance shows plateausTare called Shubnikov-de Haas oscillations. The temperature dependence of the oscillation minima is an accurate determination of the mobility and mean free path of the 2DEG.

Table 1.1. Useful relations and system parameters (including spin degeneracy). We have chosen typical values for $\mu_{\mathrm{e}} \Gamma$ and $n_{\mathrm{s}} \Gamma$ from which the other values are deduced.

$\begin{array}{ll}\text { - electron mobility } & \mu_{\mathrm{e}}=10^{6} \mathrm{~cm}^{2} / \mathrm{Vs} \text { (typical value) } \\ \text { - scattering time } & \tau=m^{*} \mu_{\mathrm{e}} / \mathrm{e}=38 \mathrm{ps} \\ \text { - effective mass } & m^{*}=0.067 m_{0} \\ \text { - electron density } & n_{\mathrm{s}}=2.810^{15} \mathrm{~m}^{-2}(\text { typical value) } \\ \text { - Fermi energy } & E_{\mathrm{F}}=\pi \hbar^{2} n_{\mathrm{s}} / m^{*}=10 \mathrm{meV} \\ \text { - Fermi velocity } & v_{\mathrm{F}}=\left(2 E_{\mathrm{F}} / \mathrm{m}^{*}\right)^{1 / 2}=2.310^{5} \mathrm{~m} / \mathrm{s} \\ \text { - Fermi wavelength } & \lambda_{\mathrm{F}}=\left(2 \pi / n_{\mathrm{s}}\right)^{1 / 2}=47 \mathrm{~nm} \\ \text { - elastic mean free path } & l=v_{\mathrm{F}} \tau=8.7 \mu \mathrm{m} \\ \text { - cyclotron radius at } E_{\mathrm{F}} & r_{\mathrm{c}}=m^{*} v_{\mathrm{F}} / e B=88 \mathrm{~nm} \text { at } B=1 \mathrm{~T} \\ \text { - angular cyclotron frequency } & \omega_{\mathrm{c}}=e B / m^{*}=2.610^{12} \mathrm{rad} / \mathrm{s} \\ \text { - magnetic length } & l_{B}=(\hbar / e B)^{1 / 2}=8.1 \mathrm{~nm} \mathrm{at} B=10 \mathrm{~T}\end{array}$

\subsection{E-BEAM FABRICATION OF A SUBMICRON SEMICONDUCTOR DEVICE}

We briefly outline a standard procedure for fabricating small devices in a 2DEG. We start from a 2DEG confined in a GaAs/AlGaAs heterostructure. To laterally confine the electrons one must define a pattern on top of the heterostructure. As an exampleTwe describe e-beam lithographyTwhich is a technique also used in the fabrication of chips. Depending on the desired pattern Tone can choose between many variations in the lithography process steps. One procedure is shown schematically in Fig. 3. An organic resist film $(\approx 100 \mathrm{~nm}$ thick) is spun onto the substrate. Exposing the resist with an electron beam results in a molecular-mass difference between the exposed and unexposed parts (see Fig. 3a). An appropriate developer removes only the exposed resist $\Gamma$ resulting in the mask pattern shown in Fig. $3 \mathrm{~b}$. Evaporated material now sticks only at the substrate where the resist has been removed (see Fig. 3c). The mask itself can be removed by dissolving the remaining resist (lift-off) leaving a small pattern on top of the substrate (see Fig. 3d). The minimum resolution of such a pattern with present day electron-beam lithography facilities is about $20 \mathrm{~nm}$. 


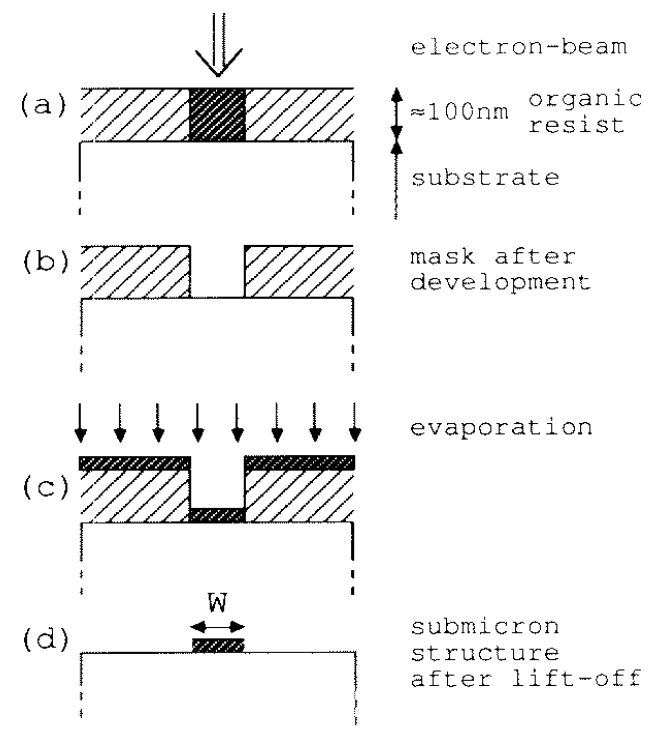

Figure 3. Outline of the electron-beam lithography procedure for fabricating submicron structures.

(a) Cross-section

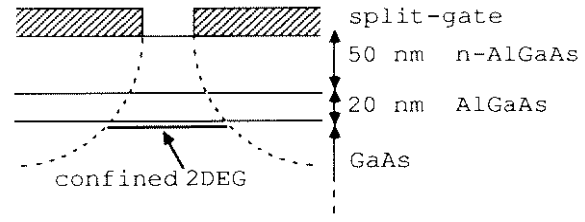

(b) Top-view

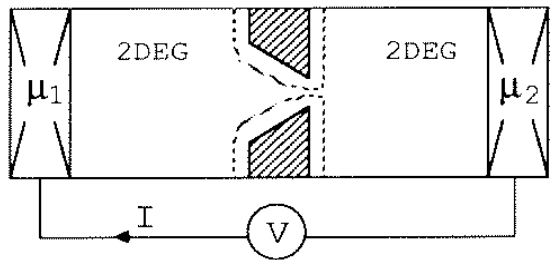

Figure 4. (a) Cross-section of a GaAs/AlGaAs heterostructure with typical layer thicknesses. A negative voltage $V_{\mathrm{G}}$ applied to the metal split-gate confines the electrons laterally in the $2 \mathrm{DEG}$.

(b) Top-view of a QPC. The dotted line indicates the depletion region in the 2DEGT which is tuned by $V_{\mathrm{G}}$. The two wide $2 \mathrm{DEG}$ regions act as reservoirs emitting electrons through the QPC with energies up to their electrochemical potentials $\mu_{1}$ and $\mu_{2}$. A voltage difference $V=\left(\mu_{1}-\mu_{2}\right) / e$ results in a net current $I$ through the QPC.

From this point there are basically two ways to transfer the pattern to the 2DEG. One way is to use the pattern as an etch mask. Etching removes the portion of the 2DEG not protected by the pattern. The boundaries of the etched pattern cause a depletion region $\Gamma$ such that the conducting width in the 2DEG is unknown and often much smaller than the defined width [2]. 
Another Tmore flexible way to transfer the pattern to the 2DEG is to use it as a gate [3Г4]. Applying a negative voltage to the gate depletes the electron gas beneath it Tthereby confining electron motion to the ungated region. For a split-gate geometryTshown in Fig. 4 This results in a narrow conducting channel. The advantage of the split-gate technique is that the conducting width of the point contact in the 2DEG can be tuned from the defined lithographic width of the pattern to zeroTby making the gate voltage more negative. Transport between the two wide 2DEG regions in Fig. 4b occurs only through the point contact and can be studied as a function of the width by changing the gate voltage. The width $W$ of the constriction can be made comparable to the Fermi wavelength $\Gamma$ so this device is called a quantum point contact (QPC). The actual induced potential in the 2DEG is unknown Tbut self-consistent calculations [5] indicate that it has a saddleshape (see Fig. 5a). In the constrictionTelectrons are confined in the lateral $x$-direction and slowed down by the presence of a potential barrier in the $y$-direction. Making the gate voltage more negative 5 simultaneously reduces the width and increases the barrier height. For zero-width or a barrier which is higher than the Fermi energy $E_{\mathrm{F}}$ of the 2DEGTthe QPC is pinched-off and electron transport between the wide 2DEG regions is impossible. Fig. $5 \mathrm{~b}$ shows a scanning electron micrograph of a double point contact device.

(a)

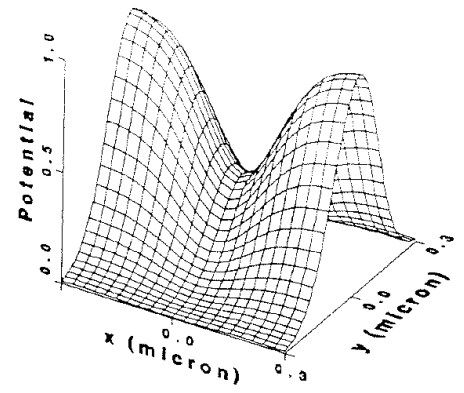

(b)

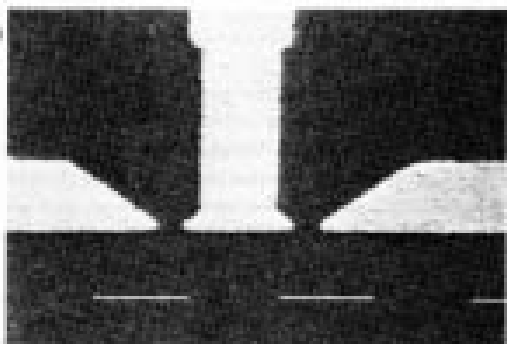

Figure 5. (a) Saddle-shaped potential induced in the 2DEG upon application of a negative gate voltage $\Gamma$ resulting in lateral confinement in the $x$-direction and a potential barrier in the longitudinal $y$-direction (from Beenakker $\Gamma$ and van Houten in Ref. [6]).

(b) Scanning electron micrograph of a double-QPC device. The white areas are the Au gates $\Gamma$ and the marker is $1 \mu \mathrm{m}$ long. The QPCs are $250 \mathrm{~nm}$ wide and are separated by $1.5 \mu \mathrm{m}$.

\subsection{QUANTIZED CONDUCTANCE OF A POINT CONTACT}

The resistance of a point contact in the classical ballistic regime is known as the Sharvin resistance [7]. The Sharvin resistance is entirely due to elastic backscattering at the geometrical narrowing of the ballistic point contact. 
Dissipative processes which bring the electron system into thermodynamic equilibrium take place far away from the point contact (i.e. several times the inelastic mean free path). Therefore $\Gamma$ the cause of the resistance in this system is spatially separated from its corresponding Joule heating. The classical conductance of a Sharvin point contact in a 2DEG is [8]

$$
G_{\mathrm{s}}=\frac{e^{2}}{\pi} \frac{\mathrm{d} N^{2 \mathrm{D}}}{\mathrm{d} E} v_{\mathrm{F}} W .
$$

The quantum mechanical 2D density of states per unit area Tincluding a factor 2 for spin degeneracy is $\mathrm{d} N^{2 \mathrm{D}} / \mathrm{d} E=m^{*} / \pi \hbar \Gamma$ while $v_{\mathrm{F}}=\hbar k_{\mathrm{F}} / m^{*}$ is the Fermi velocity. We can rewrite the classical equation (1) to a semiclassical version which includes the conductance quantum $2 e^{2} / h$

$$
G_{\mathrm{s}}=\frac{2 e^{2}}{h} \frac{k_{\mathrm{F}} W}{\pi}=\frac{2 e^{2}}{h} \frac{2 W}{\lambda_{\mathrm{F}}} .
$$

The Fermi wave-vector $k_{\mathrm{F}}$ or wavelength $\lambda_{\mathrm{F}}$ are related to the $2 \mathrm{D}$ electron density $n_{\mathrm{s}}$ by $k_{\mathrm{F}}=2 \pi / \lambda_{\mathrm{F}}=\left(2 \pi n_{\mathrm{s}}\right)^{1 / 2}$. The semi-classical form of the Sharvin conductance is continuous and linear in the width $W$. However $\Gamma$ Eq. 2 suggests that we can expect deviations due to the wave nature of electrons whenever $\lambda_{F}$ is of order $W$. We give a derivation of the quantum version of the Sharvin conductance below Tbut first discuss the experimental results.

The conductance of a point contact is measured by passing a current $I$ through the sample and measuring the voltage $V$ between the current source and drain (see Fig. 4b). Fig. 6 shows the conductance $G$ in units of $2 e^{2} / h=(12906 \Omega)^{-1}$ versus gate voltage $V_{\mathrm{G}}$ measured at $B=0$. Assuming that the width varies linear with gate voltage $\mathrm{W}$ we indeed see that on average the conductance decreases linearly when the constriction is narrowed. However $\Gamma$ around this classical dependence we see that $G$ changes in quantized steps of $2 e^{2} / h$. At $V_{\mathrm{G}}=-2.2 \mathrm{~V} \Gamma$ the conductance becomes zero $\Gamma$ corresponding to a pinched-off point contact. Fig. 6 further shows that on increasing the temperature the conductance quantization gradually disappears [9]. The conductance $G\left(V_{\mathrm{G}}\right)$ is roughly linear at $4.2 \mathrm{~K}$ Tin accordance with the classical dependence of Eq. 1. Although the classical result cannot explain the quantization $\Gamma$ we note that the plateau values are obtained in Eq. 2 whenever the width $W$ is an integer multiple of $\lambda_{\mathrm{F}} / 2$. Eq. 2 predicts that an increase in $W$ of $\lambda_{\mathrm{F}} / 2$ (which is $21 \mathrm{~nm}$ in this sample) increases $G$ by $2 e^{2} / h$. In total 16 steps were observed between pinch-off and $V_{\mathrm{G}}=-0.3 \mathrm{~V}$ were the constriction is just formed in the 2DEG. The 16 steps give an estimate of the width $W$ of about $340 \mathrm{~nm}$ Tsomewhat larger than the lithographic width of $250 \mathrm{~nm}$ Tbut consistent with the schematic depletion profile shown in Fig. 4(a). These considerations are reminiscent 


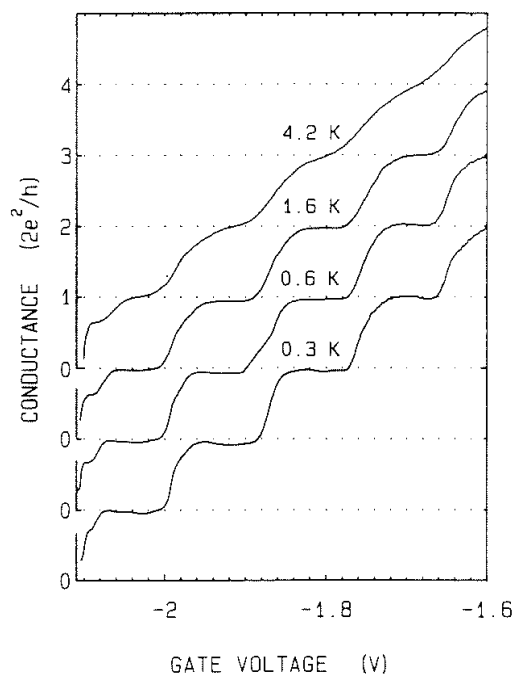

Figure 6. Conductance versus gate voltage at $B=0$ and different temperatures. Increasing the temperature thermally averages the higher plateaus first (from Ref. [9]).

of the states of a particle-in-a-1D-box Twhich Tas we show below $\Gamma$ is the basic idea behind the conductance quantization.

We note that the conductance quantization is not as exact as the quantum Hall effect. First $\Gamma$ a series resistance $(\approx 100 \Omega)$ originating from the wide 2DEG regions has been subtracted to line up the plateaus at their quantized values [8]. Furthermore 5 the plateaus are not completely flat. This may be due to scattering at impurities in the vicinity of the QPC or $\Gamma$ as we discuss below Tthe abruptness of the constriction.

We now discuss that the conductance quantization for transport through 1D subbands. If the potential which describes the transition from the wide 2DEG regions to the narrowest point in the QPC varies sufficiently smoothly (i.e. adiabatically) Tthe potential variation in the $x$ - and $y$-directions may be decoupled [10] (see Fig. 5a). The narrowest point forms the bottleneck of the QPC in the sense that it completely determines the transport properties. In this case we can calculate transport through a QPC from the Hamiltonian

$$
H=\frac{p_{x}^{2}}{2 m^{*}}+e V(x)+\frac{p_{y}^{2}}{2 m^{*}},
$$

where $V(x)$ describe the transport at the bottleneck. For the confinement in the lateral $x$-direction $\Gamma$ we follow Berggren et al. [11] and choose a parabolic confining potential $V(x)=1 / 2 m^{*} w_{0}^{2} x^{2}$. Self-consistent calculations of Laux et al. [5] have shown that small split-gate samples have a confinement close to such a parabola. The advantage of using a parabolic potential is that the 
resulting Schrödinger equation can be written in the form of a harmonic oscillator having energy eigenvalues

$$
E_{n}=\left(n-\frac{1}{2}\right) \hbar \omega_{0}+\frac{\hbar^{2} k_{y}^{2}}{2 m^{*}} \quad(n=1,2, \ldots)
$$

which contains a free-electron kinetic energy dispersion in the longitudinal $y$-direction. In the lateral $x$-direction the energy statesTindexed by $n=$ $1,2, \ldots$ Tare quantized and separated in energy by $\hbar \omega_{0}$.

Because the electron motion is free in one direction onlyГEq. 4 describes 1D subbands. Fig. 7 shows the 1D subband dispersion versus longitudinal wave-vector $k_{y}$. The right-going electrons $\Gamma$ with a velocity $\hbar v_{n}=\mathrm{d} E_{n} / \mathrm{d} k_{y} \Gamma$ originate from the left $2 \mathrm{DEG}$ reservoir Wwhich at zero temperature populates all the states up to its electrochemical potential $\mu_{1}$. SimilarlyГthe left-going electron states are occupied up to $\mu_{2} \Gamma$ the electrochemical potential of the right 2DEG reservoir (see Fig. $4 \mathrm{~b}$ ).

A voltage difference $V=\left(\mu_{1}-\mu_{2}\right) / e$ between the two reservoirs results in a net current $I \Gamma$ which is carried by the (uncompensated) electron states in the energy interval between $\mu_{1}$ and $\mu_{2}$. Note that we define the Fermi energy as $E_{\mathrm{F}}=\mu_{1}=\mu_{2}$ when $V=0$. The net current $I$ at zero temperature is

$$
I=e \sum_{n=1}^{N} \int_{\mu_{2}}^{\mu_{1}} \mathrm{~d} E \frac{1}{2} \frac{\mathrm{d} N_{n}}{\mathrm{~d} E} v_{n}(E) T_{n}(E),
$$

which includes the transmission probability of the $n$-th subband $T_{n}(E)$ to describe possible scattering events. Here $N$ denotes the number of occupied subbands $\Gamma$ i.e. the largest number for which $E_{N}\left(k_{y}=0\right)<E_{\mathrm{F}}$. The 1D spindegenerate density of states is $\mathrm{d} N_{n} / \mathrm{d} E=2 / \pi\left(\mathrm{d} E_{n} / \mathrm{d} k_{y}\right)^{-1}$. The important aspect of 1D transport is the cancelation of the energy dependence in the product of velocity and density of states $\left(\mathrm{d} N_{n} / \mathrm{d} E\right) v_{n}=4 / h$. For small voltages $\left(e V \ll E_{\mathrm{F}}\right) \Gamma$ one can take $T_{n}(E)=T_{n}\left(E_{\mathrm{F}}\right)$. Substituting this in Eq. 5Tone finds that the conductance $G=I / V=e I /\left(\mu_{1}-\mu_{2}\right)$ is independent of energy

$$
G=\frac{2 e^{2}}{h} \sum_{n=1}^{N} T_{n}\left(E_{\mathrm{F}}\right) .
$$

Eq. 6 is known as the 2-terminal Landauer formula [12]. If no backscattering takes place $\Gamma$ so that $\sum_{n=1}^{N} T_{n}\left(E_{\mathrm{F}}\right)=N \Gamma E q .6$ reduces to

$$
G=\frac{2 e^{2}}{h} N
$$




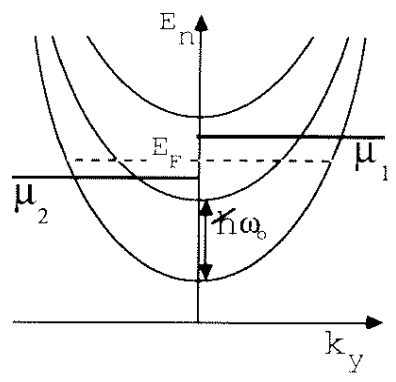

Figure 7. Energy $E_{n}$ versus longitudinal wave-vector $k_{y}$ from Eq. 4 at the bottleneck of a QPC assuming a parabolic confinement potential. The $1 \mathrm{D}$ subbands are separated by $\hbar \omega_{0}$. A net current results from the uncompensated occupied electron states in the interval between $\mu_{1}$ and $\mu_{2}$ Tthe electrochemical potentials of the two wide $2 \mathrm{DEG}$ reservoirs.

demonstrating that each occupied subband contributes $2 e^{2} / h$ to the conductance. The subbands are called 1D current channels to emphasize that each channel carries the same amount of current.

In the experiment $\Gamma$ a decreasing $V_{\mathrm{G}}$ increases the barrier in the $\mathrm{QPC} \Gamma$ and simultaneously increases the lateral confinement and consequently the energy splitting. Both effects increase the subband energies. As long as $E_{\mathrm{F}}$ is between two subband bottoms $\Gamma N$ is constant and $G$ is quantized. If a subband bottom moves through $E_{\mathrm{F}} \Gamma N$ changes by 1 and $G$ by $2 e^{2} / h$.

Several numerical calculations [13] have shown that Eq. 7 gives an accurate description of a QPC with the assumptions that impurity scattering is absent and that the potential variations are smooth. Sharp potential variations possibly present at the entrance and exit of the QPC [14] or originating from impurities [15Г 16] can give rise to backscattering and therefore destroy the quantization. The assumption of an adiabatic constriction in Eq. 3 is therefore no longer valid. At $T>0$ the reservoirs inject electrons with a Fermi-Dirac distribution $\Gamma$ which averages the conductance $G(T)=\int \mathrm{d} E(-\mathrm{d} f / \mathrm{d} E) G(E)$. Comparing this with the temperature dependence of the curves in Fig. 6 Tit is found that the subband separation gradwally increases from about $1 \mathrm{meV}$ at $V_{\mathrm{G}}=-1 \mathrm{~V}$ to $3 \mathrm{meV}$ at $V_{\mathrm{G}}=-2.1 \mathrm{~V}$. This illustrates that thermal averaging has a stronger effect on the higher plateaus Tas observed in Fig. 6.

\subsection{DEPOPULATION OF 1D MAGNETO-ELECTRIC SUBBANDS}

We now turn to the case of a QPC with an applied magnetic field in the z-direction. In the Hamiltonian of Eq. 3T the magnetic field $B$ is included by substituting $(\mathbf{p}-\boldsymbol{e A})$ for the momentum $\mathbf{p}$. In the Landau gauge for the vector potential $\mathbf{A}=A_{y}=B x$ Tthe Schrödinger equation is once again 


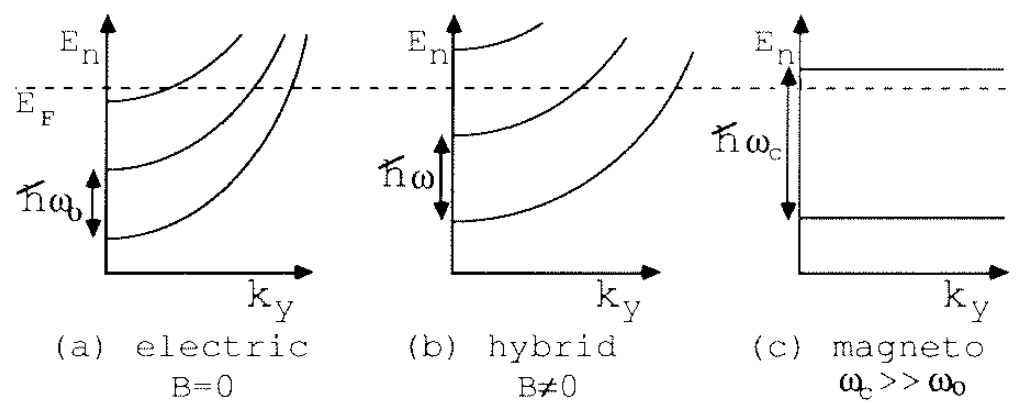

Figure 8. 1D subband dispersion for three values of the magnetic field $\Gamma$ illustrating magnetic depopulation. The energy splitting is electric $\left(\hbar \omega_{0}\right)$ in (a) $\Gamma$ and hybrid ( $\hbar \omega \Gamma$ with $\omega^{2}=\omega_{0}^{2}+\omega_{c}^{2}$ ) in (b). For large magnetic fields (c) the 1D subbands are Landau levels with an energy splitting of $\hbar \omega_{c}$.

that of a harmonic oscillatorTbut now with energy eigenvalues [11]

$$
E_{n}=\left(n-\frac{1}{2}\right) \hbar \omega+\frac{\hbar^{2} k_{y}^{2}}{2 m_{\mathrm{B}}}
$$

These eigenvalues describe hybrid magneto-electric subbands. With $\omega^{2}=$ $\omega_{0}^{2}+\omega_{\mathrm{c}}^{2} \Gamma$ where the cyclotron frequency is $\omega_{\mathrm{c}}=e B / \mathrm{m}^{*} \Gamma$ the energy separation is now a combination of the electrical confinement and the magnetic field. $m_{B}=m^{*} \omega^{2} / \omega_{0}^{2}$ is a magnetic field dependent effective mass yielding a smaller dispersion for larger magnetic fields. The influence of the magnetic field on the subband dispersion is shown schematically in Fig. 8. For $B=0$ the subbands are determined by the electrical confinement only. A small magnetic field increases the subband splitting and reduces the dispersion. For large magnetic fields $\Gamma$ the subbands have the magnetic energy separation and a vanishing dispersion. In this case $\Gamma$ the subbands are the well-known Landau levels. It can be seen from Fig. 8 that on increasing the magnetic field The number of occupied subbands decreases. This process is known as the depopulation of magneto-electric subbands.

One can show that the velocity and density of states also cancel in a magnetic field and that Eqs. ( 6) and ( 7 ) are still valid [17Г18]. From the above analysis it follows that a gradual transition exists between the quantized conductance $G=N 2 e^{2} / h$ at zero magnetic field (with $N$ the number of occupied electric subbands in Fig. 8a) to the quantum Hall conductance $G_{\mathrm{H}}=N_{\mathrm{L}} 2 e^{2} / h$ at a high magnetic field (with $N_{\mathrm{L}}$ the number of occupied Landau levels in Fig. 8c). We note that our particular choice of a parabolic confinement does not affect the general conclusions $\Gamma$ such as the cancelation of velocity with the density of statesTand the conductance quantization at zero and non-zero magnetic field. 


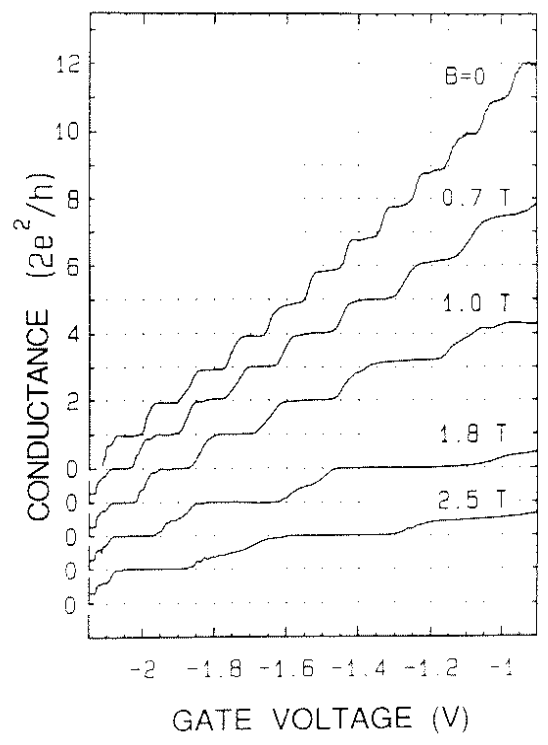

Figure 9. QPC conductance versus gate voltage at $0.6 \mathrm{~K}$ for several values of magnetic field. The increasing width of the plateaus demonstrate the increasing energy splitting in a magnetic field. The curves have been offset for clarity (from Ref. [18]).

Fig. 9 shows the conductance of a QPC versus gate voltage for several values of the magnetic field [18]. As can be seen $\Gamma$ the quantization is preserved in a magnetic field. Above $B=1$ TTspin-resolved plateaus develop at odd multiples of $e^{2} / h$. The depopulation can be seen from the fact that at a fixed gate voltage $\mathrm{Tthe}$ number of plateaus (orTequivalently the number of occupied subbands) $\Gamma$ decreases with increasing magnetic field. From the measurements of Fig. 9Гone can deduce subband splittings of about $1 \mathrm{meV}$ at $V_{\mathrm{G}}=-1 \mathrm{~V}$ and $3 \mathrm{meV}$ at $V_{\mathrm{G}}=-2 \mathrm{~V} \Gamma$ in agreement with the values obtained from the temperature dependence [9]. A third independent way to determine the subband splittings is by measuring the non-linear current-voltage characteristics of a QPC [19].

\subsection{ELECTRON TRAJECTORIES IN A LOW MAGNETIC FIELD}

In the previous Section $\Gamma$ we discussed the influence of a magnetic field on the subband dispersion in momentum space. We now consider the electron motion in real space $\Gamma$ which yields a simple physical picture of the QHE and associated effects. To elucidate the quantized electron motion in a high magnetic field $\Gamma$ we first discuss classical electron trajectories in a low magnetic field. 

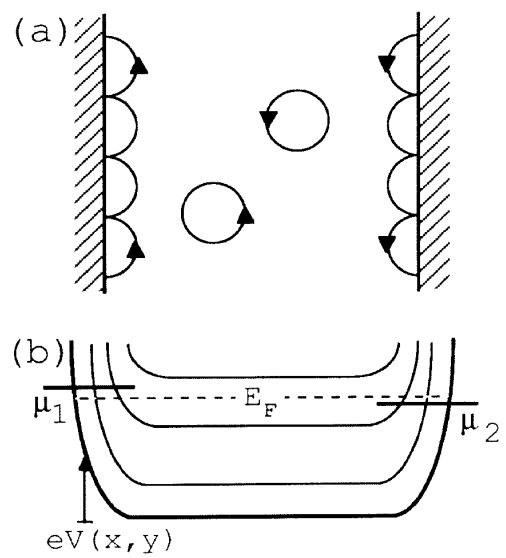

Figure 10. (a) Schematic classical electron trajectories in a magnetic field.

(b) Corresponding quantum picture of the energy states of Eq. (10) along a cross-section of the 2DEGTillustrating the formation of edge channels at the boundary of the 2DEG (formed by the electrostatic potential energy $\mathrm{eV}(x, y)$ ) where the Landau levels intersect the Fermi energy $E_{\mathrm{F}}$.

In the absence of an electric field $E$ T the balance of the Lorentz force $F_{\mathrm{L}}=e v B$ and the centripetal force $F=m^{*} v^{2} / r$ leads to a cyclotron motion of the electrons $\Gamma$ with an angular frequency $\omega_{c}=e B / m^{*}$ and at the Fermi energy a cyclotron radius $r_{\mathrm{C}}=m^{*} v_{\mathrm{F}} / e B$ (see Fig. 10a). When the electric field $E=-\nabla V(x, y) \neq 0$ Tthe electrons have a net drift velocity $v_{\mathrm{D}}=E / B$. At the boundary of the sample $\Gamma$ where $E$ is large $\Gamma$ the collisions at the edge result in skipping orbits. The electrons skip with the drift velocity along the edge of the sample. The direction of the velocity is opposite for the two opposite edges (see Fig. 10a).

The skipping orbit motion of electrons along a 2DEG boundary in a small magnetic field has been observed in an electron focusing experiment by van Houten et al. [20]. The geometry shown in Fig. 5b with two adjacent QPCs with a separation of $L=3 \mu \mathrm{m}$ was used $\Gamma$ where one QPC injects electrons into the 2DEG and the second QPC collects them. The injected electrons are focused by the magnetic field on the boundary between injector and collector at distances $2 p r_{\mathrm{c}}(p=1,2, \ldots)$. Focusing into the collector occurs when $2 p r_{c}=L$. In the experiment the collector voltage is measured as a function of magnetic field $B$. From the condition $2 p r_{c}=L$ it follows that the largest number of electrons reaches the collector when $B_{\text {foc }}=2 p m^{*} v_{\mathrm{F}} / e L$ which leads to periodic oscillations in the collector signal. This is shown in Fig. 11 together with a calculation. On average the voltage increases linearly with magnetic field $\Gamma$ which is expected for the classical Hall resistance $V_{\text {Hall }} / I=B / e n_{\mathrm{s}}$. For curve (a) $\Gamma$ however $\Gamma$ we see 


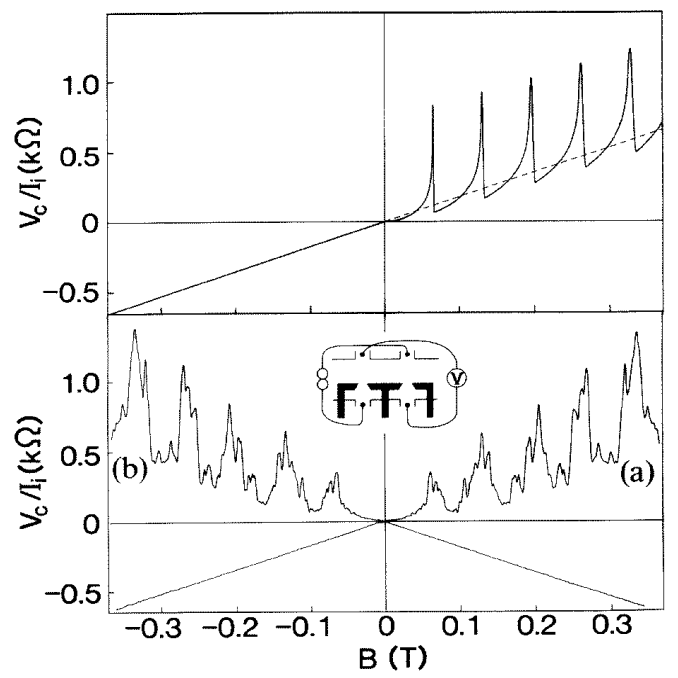

Figure 11. Bottom: Electron focusing $(T=50 \mathrm{mK} \Gamma L=3.0 \mu \mathrm{m})$ in the configuration depicted in the inset. The two traces $a$ and $b$ are measured with interchanged current and voltage leads $\Gamma$ and demonstrate the injector-collector reciprocity as well as the reproducibility of the fine structure. Top: Calculated classical focusing spectrum corresponding to the experimental trace a $(50 \mathrm{~nm}$ wide point contacts were assumed). The dashed line is the extrapolation of the classical Hall resistance seen in reverse fields. (From Ref. [20].)

additional large oscillations with fine structure around the average Hall resistance for positive magnetic field. These oscillations occur at the expected focusing fields. For a negative magnetic field no focusing signal is observed since now the injected electrons are deflected away from the collector.

Curve (b) is taken with the current and voltage probes interchanged. The relation between (a) and (b) demonstrates Büttiker's reciprocity relation $[21] \Gamma$

$$
R_{i j, k l}(B)=R_{k l, i j}(-B) .
$$

It implies that upon interchange of the current probes $i$ and $j$ with the voltage probes $k$ and $l \Gamma$ one obtains the same resistance at the opposite magnetic field. This fundamental symmetry relation is clearly confirmed in Fig. 11Tincluding the symmetry in the fine-structure. The latter has been explained by extending the classical focusing calculation of the top section to include quantum interference effects between trajectories being injected at different angles [20]. This focusing experiment demonstrates that the collisions at the boundary between injector and collector are highly specular $\Gamma$ since diffusive boundary scattering would average the oscillations. In a subsequent experiment by Spector et al. [22] focusing signals were observed up to a distance between injector and detector as large as $64 \mu \mathrm{m}$. 


\subsection{THE QUANTUM HALL EFFECT ON MESOSCOPIC LENGTH SCALES}

\subsubsection{Edge Channels}

In a high magnetic field the electron motion is quantized. The flux $\Phi$ enclosed by an electron in a cyclotron orbit equals an integer times the flux quantum $\Phi_{0}=h / e$ Tand the quantized electron energies are:

$$
E_{n}=\left(n-\frac{1}{2}\right) \hbar \omega_{c}+e V(x, y)
$$

where $n=1,2, \ldots$ is the spin-degenerate Landau level index $\Gamma$ and we have ignored the Zeeman energy splitting $\pm g \mu_{\mathrm{B}} B / 2$. We assume that the electrostatic potential $V(x, y)$ is flat in the interior of the sample and rises at the boundary. Electrostatic variations due to impurities are ignored $\Gamma$ because we are dealing with ballistic samples. Fig. 10b shows schematically the Landau energy levels of Eq. 10. The electron states at the left boundary are occupied up to $\mu_{1} \Gamma$ the electrochemical potential of the current source $\Gamma$ and at the right boundary up to $\mu_{2} \Gamma$ the electrochemical potential of the current sink (see Fig. 4b). At the two sample boundaries Tthe electron states have opposite velocity directions Tsimilar to the classical case of Fig. 10a.

The relevant electron states for linear transport are only those at the Fermi energy. As can be seen $\Gamma$ these are located at the sample boundaries $\Gamma$ where the Landau levels intersect the Fermi energy $\left(E_{n}=E_{\mathrm{F}}\right)$ Tand they extend in the direction perpendicular to the cross-section of Fig. 10b. The intersections are the current-carrying states $\Gamma$ which are known as edge channels [17Г 23Г 24]. The net current $I$ only results from the uncompensated states in the interval between $\mu_{1}$ and $\mu_{2}$. The total current carried by the states below $\mu_{2}$ is zero. The transport through edge channels is 1dimensional [17]. Edge channels can therefore also be viewed as 1D current channels $\Gamma$ each carrying a current $I_{n}=2 e / h\left(\mu_{1}-\mu_{2}\right)$. With the Hall voltage $V_{\mathrm{H}}=\left(\mu_{1}-\mu_{2}\right) / e$ measured between the two sample boundaries $\Gamma$ this directly gives the quantized Hall conductance $G_{\mathrm{H}}=N_{\mathrm{L}} I_{n} / V_{\mathrm{H}}=N_{\mathrm{L}} 2 e^{2} / h$.

Up to now we have ignored all scattering processes. Büttiker [25] has pointed out that due to the spatial separation of the electron states with opposite velocity backscattering requires scattering from one sample boundary to the other. Backscattering is therefore suppressed when the edge states between $\mu_{1}$ and $\mu_{2}$ are not connected by extended electron states. This is the case in Fig. $10 \mathrm{~b}$ एwhere the Fermi energy is between two bulk Landau levels.

\subsubsection{Selective Probing of Edge Channels}

The above description of transport in the quantum Hall effect (QHE) regime known as the Landauer-Büttiker formalism $\Gamma$ is reviewed in Ref. [26]. This edge channel description gives an appealing physical picture of the 
QHE. The question now arises about how to prove the existence of edge channels and whether they can really be viewed as independent current channels. We now discuss an experiment involving two adjacent QPCs which directly probes the transport through a particular edge channel. The theory for this experiment provides a simple illustration of Biittiker's multiprobe formalism which has been used very successfully to describe different kind of mesoscopic transport phenomena.

In Section 1.5 we discussed the electron focusing from one QPC to an adjacent second QPC by small magnetic fields $(B \leq 1 \mathrm{~T})$. These fields are too small to quantize the electron motion Tand the focusing can be explained in terms of classical cyclotron motion. We consider now the same geometry in the high field regime $(B \geq 1 \mathrm{~T})$. Fig. 12a shows schematically two adjacent QPCs $A$ and $B$ defined in a 2DEG with an applied magnetic field such that two edge channels are occupied. The 2-terminal conductances $G_{\mathrm{A}}$ and $G_{\mathrm{B}}$ of the individual QPCs measure the number of transmitted channels and are quantized in multiples of $2 e^{2} / h$ (see also Fig. 9). The Hall conductance $G_{\mathrm{H}}$ is normally thought to be independent of the characteristics of the current and voltage probes and to correspond directly to the number of occupied (spin-degenerate) Landau levels $N_{\mathrm{L}}$ in the $2 \mathrm{DEG} G_{\mathrm{H}}=N_{\mathrm{L}} 2 e^{2} / h$. This is not true for the situation in Fig. 12a and we discuss here that for describing transport through mesoscopic conductors one has to include the properties of the measurement contacts.
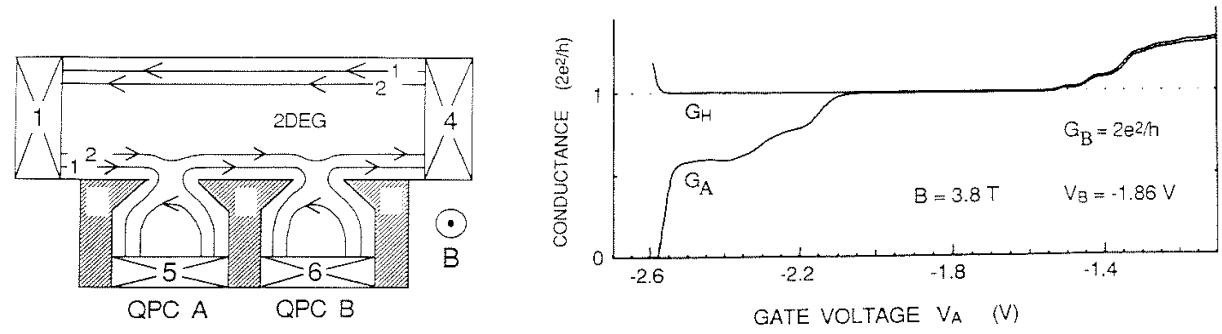

Figure 12. Left: Geometry used to observe an anomalous quantization of the Hall conductance $\Gamma$ for which QPC $A$ is used as current probe and QPC $B$ as voltage probe. Right: Comparison between the Hall conductance $G_{\mathrm{H}}$ and the 2-terminal conductance of the current probe $G_{\mathrm{A}}$. The voltage probe conductance is kept fixed at $G_{\mathrm{B}}=2 e^{2} / h$. Although the number of occupied Landau levels in the $2 \mathrm{DEG}$ is unchanged $\Gamma G_{\mathrm{H}}$ follows the largest probe conductance (from Ref. [27])

We first assume that the edge channels shown in Fig. 12a are independentГi.e. no scattering events occur between different edge channels or $\Gamma$ equivalently electrons travel with conservation of quantum-subbandnumber. In this case the transport is adiabatic. The edge channels are oc- 
cupied up to the electrochemical potential of the last Ohmic contact they have left. (We assume ideal Ohmic contacts $\Gamma$ meaning that all incoming electrons are absorbed and all outgoing states are occupied up to an average electrochemical potential.) Consequently in the region between the two QPCsT the two edge channels have an unequal population. Channel-2 is occupied up to $\mu_{1}$ Twhile current probe 5 populates channel- 1 up to $\mu_{5}$. This process is called selective population of edge channels. If the voltage probe $B$ detects all edge channels like an ideal Ohmic contact Tthe regular quantum Hall conductance is measured. However Tthis is not the case in Fig. 12a where the voltage probe selectively detects only the first channel. The second channel is neither populated by the current probe nor detected by the voltage probe and $\Gamma$ therefore $\Gamma$ is not measured. The Hall conductance $G_{54,61}$ equals $2 e^{2} / h$ instead of $4 e^{2} / h$ which a regular Hall measurement would give. The current and voltage contacts do not measure all the $2 \mathrm{DEG}$ properties $\Gamma$ but only those properties they "see" Tor couple to. In general one can show that in the absence of inter-edge channel scattering this Hall conductance is given by $[27]$

$$
G_{\mathrm{H}}=\max \left\{G_{\mathrm{A}}, G_{\mathrm{B}}\right\}
$$

implying that $G_{\mathrm{H}}$ is completely determined by the characteristics of the probes and is independent of the number of occupied Landau levels in the 2DEG.

Fig. $12 \mathrm{~b}$ compares the measured probe conductances $G_{\mathrm{A}}$ and $G_{\mathrm{B}}$ with the Hall conductance $G_{\mathrm{H}}$. The magnetic field is kept fixed at 3.8 TTcorresponding to $N_{\mathrm{L}}=2$ in the bulk 2DEG. The voltage on QPC BCdefining the voltage proberis also fixed such that only the first edge channel is transmitted $\Gamma$ and therefore $G_{\mathrm{B}}=2 e^{2} / h$. The voltage $V_{\mathrm{gA}}$ on QPC $A$ Tdefining the current probe $\Gamma$ is varied $\Gamma$ resulting in a decreasing $G_{\mathrm{A}}$. Again we note that a normal Hall measurement would give a constant conductance $G_{\mathrm{H}}=4 e^{2} / \mathrm{h}$. HoweverTFig. 12b shows a Hall conductance which virtually follows the largest probe conductance in agreement with Eq. 11 (for $V_{\mathrm{gA}}>-1.5 \mathrm{VT}$ $G_{\mathrm{H}}=G_{\mathrm{A}}>2 e^{2} / h$; for $-2.1 \mathrm{~V}<V_{\mathrm{gA}}<-1.5 \mathrm{~V} \Gamma G_{\mathrm{H}}=G_{\mathrm{A}}=G_{\mathrm{B}}=2 e^{2} / h$ corresponding to the edge channel flow of Fig. 12a; and for $V_{\mathrm{gA}}<-2.1 \mathrm{~V} \Gamma$ $\left.G_{\mathrm{H}}=G_{\mathrm{B}}=2 e^{2} / h\right)$. The experiment demonstrates that on short distances of order $\mu \mathrm{m}$ the transport through edge channels is adiabatic $\Gamma$ implying that they can be viewed as independent 1D current channels. Subsequent experiments have shown that a non-equilibrium population can persist up to larger distances (several tens of $\mu \mathrm{m}$ 's) [28]. In particularCit is found that the top most channel is virtually decoupled from the lower edge channels even over macroscopic distances of several times $100 \mu \mathrm{m}$ [28Г29Г30Г 31 Г 32$]$. These experiments have clarified the important role of measurement probes in the QHE regime. 


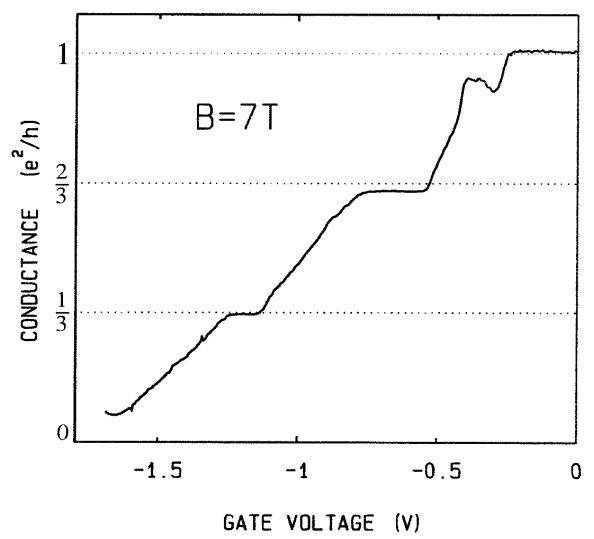

Figure 13. Two-terminal conductance of a quantum point contact at $B=7 \mathrm{~T}$. At zero gate voltage the conductance is $G=e^{2} / h$ implying that the $2 \mathrm{DEG}$ is spin-polarized at filling factor 1 . On decreasing the gate voltage fractional plateaus are observed (from Ref. [37].

1.6.3. The Importance of Electron-Electron Interactions for the QHE on Mesoscopic Length Scales

In our discussion we neglected Coulomb interactions between the electrons. For many experimental observations it is sufficient to give a description in terms of a non-interacting electron model. There are several exceptions $\Gamma$ however. First of all in very high mobility samples not only are plateaus observed at integer filling factors $\mathrm{Tbut}$ also at fractional filling factors (see Fig. 2). The theory for this fractional QHE needs to incorporate electronelectron interactions [33]. Recently $\mathrm{\Gamma}$ a new appealing formulation for the fractional QHE has been given in terms of composite particles [34]. Another recent development is the prediction [35] and observation [36] of so-called skyrmions.

Also Ton mesoscopic length scales it has recently become appreciated that electron-electron interactions can be important under certain circumstances. First of all the FQHE can also be observed at short ballistic length scales [37]. Fig. 13 shows the conductance of a QPC at $7 \mathrm{~T}$. At zero gate voltage the conductance is equal to $e^{2} / h$ showing that the filling factor of the bulk 2DEG is equal to one (i.e. spin resolved). On making the gate voltage more negative the conductance decreases. However $\Gamma$ at fractional values $2 / 3$ and $1 / 3$ times $e^{2} / h$ it shows quantized plateaus. The QPC used in this experiment shows quantized conductance at $B=0$ implying that fractional plateaus can exist in a ballistic sub-micron device [37Г38]. Also $\Gamma$ selective population and detection experiments of fractional edge channels have been performed [39Г 40] in a similar way as in Section 1.6.2 for the case of integer edge channels. These experiments on the fractional QHE in 
the ballistic regime can not be explained with the non-interacting electron models of Sections 1.6.1 and 1.6.2. Instead interacting models have been proposed by Beenakker [41] TMacDonald [42] and Chang [43]. These models are the basis of recent models on self-consistent calculations of edge channels [44]. From these electrostatic calculations it follows that edge channels

have a finite width. Regions where the Fermi energy is pinned in a Landau level are called a compressible liquid whereas regions where the Fermi energy is between Landau levels are called an incompressible liquid. Although $\Gamma$ Coulomb interactions give edge channels a finite width the selective population experiments suggest that they remain 1D channels. In 1D Coulomb interactions are known to give rise to a new type of electron liquid. This so-called Luttinger liquid is discussed by Fisher and Glazman in this volume including predictions for correlated electron transport in fractional edge channels. AltogetherTwe can conclude this Section with the statement that the QHE is still a very interesting system for studying new electronic properties.

\section{Single-electron tunneling}

\subsection{REVIEW}

The electron transport in two-dimensional electron gases displays the quantum coherence of the electronic states Tas described in the previous Section. Another important aspect of mesoscopic systems is the role of interactions. We mentioned already their importance in connection with the fractional QHE. Interaction effects also influence strongly electron tunneling through mesoscopic systems $\Gamma$ and they lead to strong correlation effects. In this Section we will discuss situations where it is sufficient to approximate the Coulomb interaction by an effective capacitance model. This applies for metal junction systems where after a relaxation of the space distribution of the electrons the remaining Coulomb interaction is well described by the geometric capacitance of the junctions. The capacitance model also works remarkably well for the transport through small quantum dots. (See the Chapter of Kouwenhoven et al. for many facts on quantum dots.) The "charging effects" allow us to control single electron charges $\Gamma$ which leads to a variety of single-electron effects $\Gamma$ e. $g$. to the suppression of tunneling $\Gamma$ a phenomenon known as the Coulomb blockade.

Single-electron effects have been studied for more than a decade $\Gamma$ and a large number of papers $\Gamma$ incl. several reviews have been devoted to the topic. Kulik and Shekhter [45] and Averin and Likharev [46] developed the perturbation theory of single-electron tunneling in metal junctions and discussed several consequences. Initial scepticism against the new concepts 
was overcome when experiments proved to be successful. After early experiments by Fulton and Dolan [47] Timportant breakthroughs were achieved in Delft by Mooij and Geerligs and in Saclay by Devoret EstèveГUrbina and further members of these groups [48Г49]. Their work is well summarized in the proceedings of an earlier NATO ASI Single Charge Tunneling [50].

In this Section we introduce the concepts and basic description of singleelectron tunneling in systems with strong charging effects. For definiteness we first consider metallic electrodes with a large density of electron states. We study how the charging energy depends on the number of electrons and the transport and gate voltages applied to various parts of the system. The simplest model systems demonstrating these features are the "singleelectron box" and the "single-electron transistor". We then derive within perturbation theory the single-electron tunneling rates. In low capacitance systems it is crucial to account for the change in the charging energy associated with the tunneling process. A master-equation description accounts for the large-scale features of the current-voltage characteristic of the singleelectron transistor. In the Coulomb-blockade regime where single-electron tunneling is suppressed Thigher-order processes such as coherent "cotunneling" of electrons through several junctions become observable. Finally we discuss single-electron tunneling through quantum dots $\Gamma$ where the level quantization becomes observable as well..

Several extensions have been described in the literature. We mention only a few:

- The mesoscopic junction systems studied here are small such that charging effects and higher-order quantum processes play a role. On the other hand $\Gamma$ they are large enough such that macroscopic current and voltage probes and sources can be coupled to the system. This makes the mesoscopic system susceptible to the influence of the electric circuit. A complete description has to include this circuit. The influence of the electrodynamic environment on single-electron tunneling has been reviewed in the article by Ingold and Nazarov in Ref. [50].

- In this introduction we study tunnel junctions with two normal-conducting electrodes $(\mathrm{NN})$. If the system or part of it is superconducting the combination of single-electron tunneling $\Gamma$ Cooper pair tunneling and Andreev reflection leads to further highly interesting effects [51]. For a review see the Chapter of Fazio and Schön in this volume.

- Many of the single-electron effects can be described within simple perturbation theory. A necessary requirement is that the resistance of the tunnel barriers is high compared to the quantum resistance $R_{\mathrm{K}}=h / e^{2} \approx 25.8 \mathrm{k} \Omega$. For tunnel junctions with lower resistance a more general formulation is needed [52Г $53 \Gamma 54]$. A systematic description of tunneling in systems with strong charging effects is presented later in the Chapter of Schoeller. 
(a)

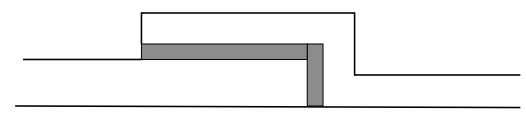

(b)

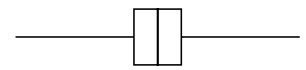

Figure 14. a) An overlap junction with an oxide layer (hatched region) $\Gamma \mathrm{b}$ ) schematic diagram for a tunnel junction.

\subsection{CHARGING ENERGY AND SINGLE-ELECTRON DEVICES}

\subsubsection{The Energy Scale}

Modern lithographic techniques allow the fabrication of narrow metallic lines with widths down to approximately $20 \mathrm{~nm}$ Tas well as tunnel junctions in overlap regions of such linesTas illustrated in Fig. 14. The structures are grown by evaporation of the metalГe. g. Aluminum $\Gamma$ through masks onto the substrate. Tunnel junctions can be produced by shadow evaporation techniques $\Gamma$ which involves two stages of evaporation at different angles. Between the two stages the first layer is allowed to oxidize. The junction is formed in the overlap region. They can be produced reliably with areas of $S=(100 \mathrm{~nm})^{2}$ and below. The oxide layer is roughly $d=10 \AA$ thick $\Gamma$ and the dielectric constant of the oxide is $\epsilon \approx 10$. Using the classical expression for the capacitance we arrive at $C=\epsilon S /(4 \pi d) \approx 10^{-15} \mathrm{~F}$.

The capacitance introduces an energy scale $\Gamma$ the charging energy corresponding to a single-electron charge $(-e) \Gamma$

$$
E_{\mathrm{C}} \equiv \frac{e^{2}}{2 C}
$$

which characterizes all charging effects. It is of the order of $E_{\mathrm{C}} \approx 10^{-4} \mathrm{eV}$ if the capacitance is $C=10^{-15} \mathrm{~F}$ Twhich corresponds to a temperature $E_{\mathrm{C}} / k_{\mathrm{B}} \approx 1 \mathrm{~K}$. In a tunneling process the electrostatic energy changes by an a mount of the order of magnitude of $E_{\mathrm{C}}$. Hence we expect in the sub-Kelvin regime electron transport to be affected by charging effects. Similar properties have been observed in semiconductor nanostructures $\Gamma$ for instance in quantum dots in 2-dimensional electron gases. The Coulomb energy in these systems again can be characterized by a capacitance which depends on the size of the dot and also may lie in the range of $10^{-15} \mathrm{~F}$ or less. Charging effects play a role in granular materials and ultimately even in molecular systems. Here the capacitance may be as low as $10^{-18}$ FTmaking single-electron tunneling observable even at room temperatures.

\subsubsection{Single-Electron Box}

We now analyze in more detail the charging energy of simple systems of tunnel junctions. It depends on the electron number in various parts of the particular system and the applied voltages. The first example is the 


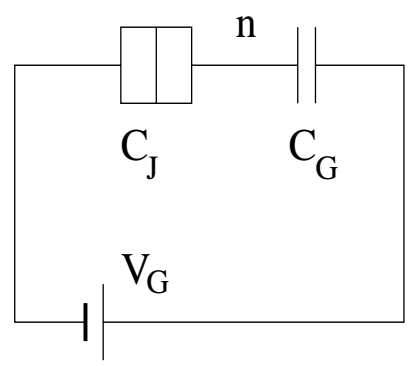

Figure 15. The single-electron box.

single-electron boxTshown schematically in Fig. 15. It consists of a small metallic island $\Gamma$ coupled via a tunnel junction with capacitance $C_{\mathrm{J}}$ to an electrode and via a capacitor $C_{\mathrm{G}}$ to a gate voltage source $V_{\mathrm{G}}$. We choose the reference such that for $V_{\mathrm{G}}=0$ the lowest energy state of the system is charge-neutral $\Gamma$ i. e. the electrons on the island compensate the charge of the ions; consequently there are $n=0$ excess electrons on the island. If a gate voltage is applied the number of excess electrons on the island can change due to tunneling across the junction in discrete steps to $n= \pm 1, \pm 2, \ldots$.

While the total number of electrons on an island is integerTthe charge is spatially distributed and in general shifted relative to the positive background. If a voltage is applied the surface charges on the capacitor plates $\Gamma$ which are of equal magnitude but opposite sign on the two sides of each junction $\Gamma$ are in general non-quantized. They are determined by the integer $n$ and the non-quantized applied voltage. We obtain the charging energy from the following elementary arguments: the total excess charge of the box splits into two parts on the left and right capacitor plate $-n e=$ $Q_{\mathrm{L}}+Q_{\mathrm{R}}$. The corresponding voltage drops add to the applied voltage $V_{\mathrm{G}}=Q_{\mathrm{L}} / C_{\mathrm{J}}-Q_{\mathrm{R}} / C_{\mathrm{G}} \Gamma$ and the charging energy is $Q_{\mathrm{L}}^{2} / 2 C_{\mathrm{J}}+Q_{\mathrm{R}}^{2} / 2 C_{\mathrm{G}}$. The relevant free energy is a Legendre transform of this energy which also contains the work done by the voltage source $-V_{\mathrm{G}} Q_{\mathrm{R}}$. Elimination of $Q_{\mathrm{L}}$ and $Q_{\mathrm{R}}$ in favor of $n$ and $V_{\mathrm{G}}$ yields $\Gamma$ up to a contribution which does not depend on the variable $n$ Tthe result

$$
E_{\mathrm{ch}}\left(n, Q_{\mathrm{G}}\right)=\frac{\left(n e-Q_{\mathrm{G}}\right)^{2}}{2 C} .
$$

Here $C=C_{\mathrm{J}}+C_{\mathrm{G}}$ is the total capacitance of the island. The effect of the voltage source is contained in the "gate charge" defined as $Q_{\mathrm{G}}=C_{\mathrm{G}} V_{\mathrm{G}}$.

The charging energy $E_{\text {ch }}$ is plotted in Fig. 16 as function of the gate charge for different excess electron numbers $n$. With increasing gate voltage $\Gamma$ the electron number corresponding to the lowest energy state increases. It does so in discrete steps from $n$ to $n+1$ at the degeneracy points $Q_{\mathrm{G}} / e=$ 


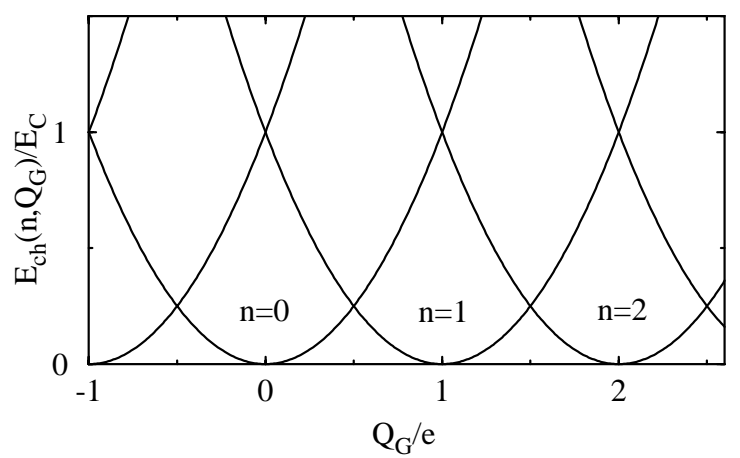

Figure 16. The charging energy of a single-electron box as a function of the gate voltage for different numbers $n$ of electron charges on the island.

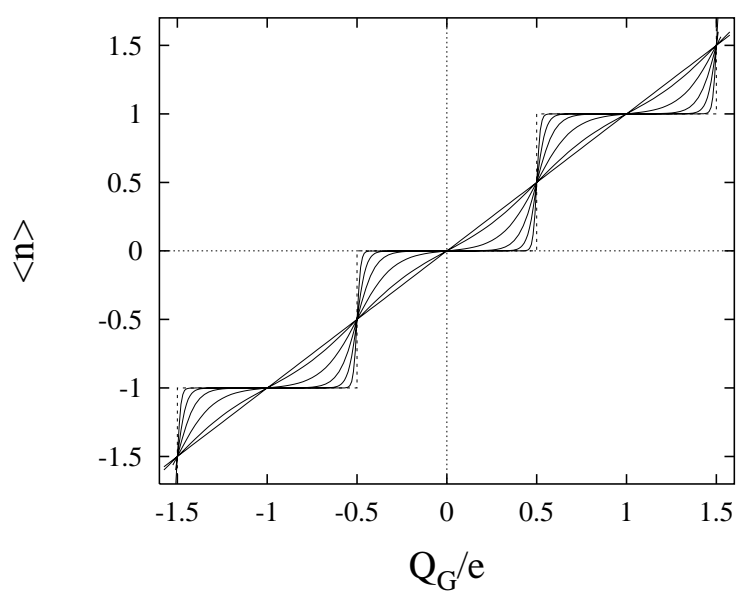

Figure 17. The average number of electron charges $\langle n\rangle$ on the island of a single-electron box as a function of the gate charge (voltage) for different temperatures $T / E_{\mathrm{C}}=0$ (dashed steps) $0.02 \Gamma 0.05 \Gamma 0.1 \Gamma 0.2 \Gamma 0.4 \Gamma$ and 1 (nearly linear).

$n+1 / 2$. Under the same conditions $\Gamma$ the voltage of the island $\Gamma V_{\text {island }}=$ $\partial E_{\mathrm{ch}} / \partial Q_{\mathrm{G}} \Gamma$ displays a sawtooth dependence on the applied voltage.

At finite temperatures the steps and sawtooth dependence are washedout Tas follows from the classical statistical average

$$
\left\langle n\left(Q_{\mathrm{G}}\right)\right\rangle=\frac{1}{Z_{\mathrm{ch}}} \sum_{n=-\infty}^{\infty} n \mathrm{e}^{-E_{\mathrm{ch}}\left(n, Q_{\mathrm{G}}\right) / k_{\mathrm{B}} T},
$$

where $Z_{\text {ch }}$ is an obvious normalization. The result is displayed in Fig. 17 for different temperatures. The stepwise increase has been observed experimentallyГe.g. by the Saclay group (see results in Ref. [50]). Their measurement 


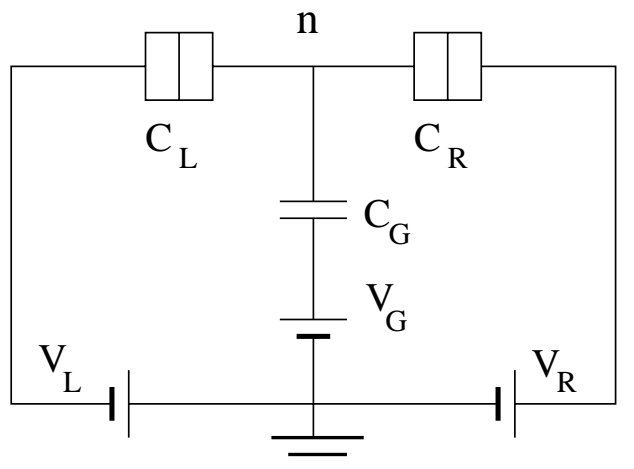

Figure 18. The SET transistor.

procedure will be discussed below. The experiments agree well with theoretical expectations. However Tit is crucial to control heating and the noise from the measurement setup which usually is at a temperature higher than that of the cryostat.

\subsubsection{Single-Electron Transistor}

Another fundamental example is provided by the single-electron transistor shown in Fig. 18. Here an island is coupled via two tunnel junctions to a transport voltage source $V=V_{\mathrm{L}}-V_{\mathrm{R}}$ such that a current can flow. The island is $\Gamma$ furthermore coupled capacitively to a gate voltage $V_{\mathrm{G}}$. The charging energy of the system depends again on the integer number of electrons $n$ on the island and the continuous voltages. Some algebra along the lines outlined for the electron box produces again $E_{\mathrm{ch}}\left(n, Q_{\mathrm{G}}\right)=(n e-$ $\left.Q_{\mathrm{G}}\right)^{2} / 2 C$. For the transistor $C=C_{\mathrm{L}}+C_{\mathrm{R}}+C_{\mathrm{G}}$ is the total capacitance of the island i.e. the sum of the two junction capacitances and the gate capacitance $\Gamma$ and all three voltage sources define the gate charge

$$
Q_{\mathrm{G}}=C_{\mathrm{G}} V_{\mathrm{G}}+C_{\mathrm{L}} V_{\mathrm{L}}+C_{\mathrm{R}} V_{\mathrm{R}} .
$$

In a tunneling process increasing the island charge from $n$ to $n+1 \Gamma$ the charging energy changes by

$$
E_{\mathrm{ch}}\left(n+1, Q_{\mathrm{G}}\right)-E_{\mathrm{ch}}\left(n, Q_{\mathrm{G}}\right)=\left(n+\frac{1}{2}-\frac{Q_{\mathrm{G}}}{e}\right) \frac{e^{2}}{C} .
$$

The second energy differences are equally spaced and can be tuned by the gate voltage. The situation is illustrated in the energy scheme shown in Fig. 19. The differences in charging energy are plotted in the center. We further display the Fermi levels of the two leads which are shifted by the applied potentials $V_{\mathrm{L} / \mathrm{R}}$. 


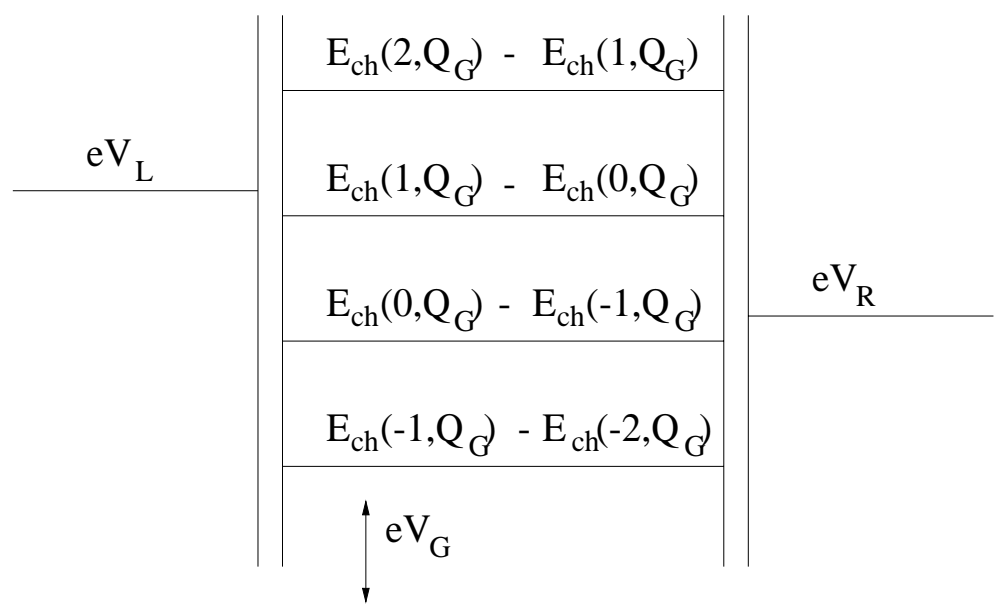

Figure 19. In the island the energy differences corresponding to the addition or removal of an electron charge are shown. They can be shifted by the gate voltage $V_{\mathrm{G}}$. The electrochemical potentials of the leads are shifted relative to each other by the transport voltage $V=V_{\mathrm{L}}-V_{\mathrm{R}}$.

For definiteness we assume that the energy of the electrons in the left lead is higher than that in the right lead. Then at low temperature tunneling from the left lead to the island (transition from $n$ to $n+1$ ) is possible if the electrochemical potential in the left lead $e V_{\mathrm{L}}$ is high enough to compensate for the increase in charging energy of the island

$$
e V_{\mathrm{L}}>E_{\mathrm{ch}}\left(n+1, Q_{\mathrm{G}}\right)-E_{\mathrm{ch}}\left(n, Q_{\mathrm{G}}\right) .
$$

Similarly tunneling from the island (transition from $n+1$ to $n$ ) to the right lead is possible at low temperature only if

$$
E_{\mathrm{ch}}\left(n+1, Q_{\mathrm{G}}\right)-E_{\mathrm{ch}}\left(n, Q_{\mathrm{G}}\right)>\epsilon V_{\mathrm{R}} .
$$

Both conditions have to be satisfied simultaneously in order for a current to flow through the transistor. It is obvious from the figure that at low transport voltages $\Gamma$ depending on the gate voltage $V_{\mathrm{G}}$ we may be either in a Coulomb blockade regime or have a finite current. By varying the gate voltage we produce the Coulomb oscillations $\Gamma$ i.e. the $\boldsymbol{e}$-periodic dependence of the conductance on $Q_{\mathrm{G}}$.

Additional devices can be examined (for a review see Estève's article in Ref. [50]): (i) The electron trap is similar to the electron box except that it contains at least two junctions in series. In contrast to the box the trap has metastable charge states. (ii) Two traps can be combined to build the electron turnstile which can serve as a current source [48]. A suitable ac-gate voltage with frequency $f$ allows the controlled transfer of 
a single-electron per cycle. Hence the current is $I=e f$. (iii) Finally in single-electron pumps a current is driven by two phase-shifted ac-voltages applied to different islands [49]. In this case a current $I=e f$ is transported even at vanishing transport voltages. The turnstile and pump can serve as a current standards $\Gamma$ if one manages to minimize the effect of missed cycles $\Gamma$ of thermal fluctuations $\Gamma$ and of quantum fluctuations. This requires low frequencies $\Gamma$ low temperatures $\Gamma$ and a design (many junctions) which minimizes higher order quantum tunneling processes.

Many properties of single-electron systems can be understood by considering only the energy of different charge configurations. However $\Gamma$ a detailed understanding of the $I-V$ characteristics requires knowledge of the tunneling rates of the electrons $\Gamma$ which will be next topic.

\subsection{TUNNELING RATES AND $I-V$ CHARACTERISTICS}

In this Subsection we introduce the Hamiltonian of the SET transistor. Using simple golden-rule arguments we derive the rate for the transfer of a single electron charge across the tunnel barriers. It depends crucially on the change in the charging energy. The transition rates enter a master equation $\Gamma$ from which we obtain the current-voltage characteristic. If a tunneling process would increase the charging energy it is suppressed at low temperature. This phenomenon is called "Coulomb blockade". This "orthodox theory" was developed in Refs. [45Г46].

\subsubsection{The Single-electron Tunneling Rate}

For definiteness we consider a SET transistor $\Gamma$ shown in Fig. $18 \Gamma$ which consists of a metallic island coupled via tunneling barriers to two leads and capacitively to an external gate voltage. Its Hamiltonian is

$$
H=H_{\mathrm{L}}+H_{\mathrm{I}}+H_{\mathrm{R}}+H_{\mathrm{ch}}+H_{\mathrm{t}} .
$$

Here $\Gamma H_{\mathrm{L}}=\sum_{k, \sigma} \epsilon_{k} c_{k, \sigma}^{\dagger} c_{k, \sigma}$ describes the noninteracting electrons with wave vector $k$ in the left lead $\Gamma$ with similar expressions for the island $H_{\mathrm{I}}$ (with states denoted by $q$ ) and the right lead $H_{\mathrm{R}}$. We allow that the leads have different electrochemical potentials. The Coulomb interaction $H_{\mathrm{ch}}$ is assumed to depend only on the total charge on the island $\Gamma$ as discussed above $\Gamma$

$$
H_{\mathrm{ch}}=\frac{\left(\hat{n} e-Q_{\mathrm{G}}\right)^{2}}{2 C} .
$$

The number operator of excess electrons on the island is given by $\hat{n}=$ $\sum_{q, \sigma} c_{q, \sigma}^{\dagger} c_{q, \sigma}-N_{+} \Gamma$ where the number of positively charged ions of the island has been subtracted. Charge transfer processes are described by the 
standard tunneling HamiltonianTfor instance tunneling in the left junction between the states $k$ and $q$ by

$$
H_{\mathrm{t}, \mathrm{L}}=\sum_{k, q, \sigma} T_{k, q} c_{k, \sigma}^{\dagger} c_{q, \sigma}+\text { h.c. } .
$$

We determine the transition rates by golden-rule arguments. The tunneling rate of an electron in the left junction is

$$
\gamma_{\mathrm{L}}^{+}(n)=\frac{1}{\epsilon^{2} R_{\mathrm{t}, \mathrm{L}}} \int_{-\infty}^{\infty} \mathrm{d} \epsilon_{k} \int_{-\infty}^{\infty} \mathrm{d} \epsilon_{q} f_{\mathrm{L}}\left(\epsilon_{k}\right)\left[1-f_{\mathrm{I}}\left(\epsilon_{q}\right)\right] \delta\left(\delta E_{\mathrm{ch}}+\epsilon_{q}-\epsilon_{k}\right) .
$$

It describes the tunneling from one of the many states $k$ in the left lead to one of the available states $q$ in the island $\Gamma$ In this process the electron number is increased from $n$ to $n+1$. The crucial point is that the energy $\Gamma$ which is conserved as expressed by the $\delta$-function $\Gamma$ contains the energies of the electron states $\epsilon_{k / q}$ Tbut also the charging energy. The latter depends on the electron number and the applied voltages $V_{\mathrm{G}}$ and $V_{\mathrm{L} / \mathrm{R}}$. In the process considered it changes by

$$
\delta E_{\mathrm{ch}}=E_{\mathrm{ch}}\left(n+1, Q_{\mathrm{G}}\right)-E_{\mathrm{ch}}\left(n, Q_{\mathrm{G}}\right)-e V_{\mathrm{L}} .
$$

We further introduced the tunnel conductance of the left junction

$$
\frac{1}{R_{\mathrm{t}, \mathrm{L}}}=\frac{4 \pi e^{2}}{\hbar} N_{\mathrm{I}}(0) \Omega_{\mathrm{I}} N_{\mathrm{L}}(0) \Omega_{\mathrm{L}}|T|^{2} .
$$

It depends on the tunnel matrix elements $T_{k, q} \Gamma$ which at this stage can be considered as constants $\Gamma$ as well as the densities of states at the Fermi level $\Gamma N_{\mathrm{I} / \mathrm{L}}(0) \Gamma$ and the volumes $\Gamma \Omega_{\mathrm{I} / \mathrm{L}} \Gamma$ of the island and lead. Equivalent expressions apply for the reverse process $\gamma_{\mathrm{L}}^{-}(n+1)$ Tdecreasing the island charge from $n+1$ to $n$ Tand for the tunneling processes in the other barriers.

In equilibrium the distribution functions $f_{\mathrm{I} / \mathrm{L}}$ are Fermi functionsTand the integrals over the electron states in Eq. (22) can be performed explicitly. The resulting "single-electron tunneling" (SET) rate is [46]

$$
\gamma_{\mathrm{L}}^{+}(n)=\frac{1}{e^{2} R_{\mathrm{t}, \mathrm{L}}} \frac{\delta E_{\mathrm{ch}}}{\exp \left[\delta E_{\mathrm{ch}} / k_{\mathrm{B}} T\right]-1} .
$$

At low temperatures $\Gamma k_{\mathrm{B}} T \ll\left|\delta E_{\mathrm{ch}}\right| \Gamma$ if the charging energy would increase in a tunneling process $\Gamma$ the tunneling is suppressed $\Gamma \gamma \rightarrow 0$. This phenomenon is called "Coulomb blockade" of electron tunneling. If charging energy is gained the rate is

$$
\gamma_{\mathrm{L}}^{+}(n)=\frac{1}{e^{2} R_{\mathrm{t}, \mathrm{L}}}\left|\delta E_{\mathrm{ch}}\right| \quad \text { for } \delta E_{\mathrm{ch}} \leq 0, T \rightarrow 0 .
$$


At finite temperatures all processes are allowed. The forward and backward rates satisfy detailed balance $\Gamma \gamma_{\mathrm{L}}^{+}(n) / \gamma_{\mathrm{L}}^{-}(n+1)=\exp \left[-\delta E_{\mathrm{ch}} / k_{\mathrm{B}} T\right]$.

A familiar limit of what is described above is a single voltage-biased tunnel junction $\Gamma$ where $\delta E_{\mathrm{ch}}$ is replaced by $-e V$ Tindependent of $n$. In this case (25) yields a linear current- voltage relation $\Gamma I_{\mathrm{t}}=e\left[\gamma^{+}-\gamma^{-}\right]=V / R_{\mathrm{t}}$. We can also reverse the argument. The two requirements - (i) a linear characteristic in the voltage-biased case and (ii) detailed balance - uniquely determine the expression for the rate to be of the form (25).

\subsubsection{Master Equation for Sequential Tunneling}

Given the electron tunneling rates we can set up a master equation for the probability $P(n, t)$ to find the island in a state with $n$ electrons. The probability changes by tunneling in the left and right junctions. Hence

$$
\begin{aligned}
\frac{\mathrm{d}}{\mathrm{d} t} P(n, t)= & -\left[\gamma_{\mathrm{L}}^{+}(n)+\gamma_{\mathrm{L}}^{-}(n)+\gamma_{\mathrm{R}}^{+}(n)+\gamma_{\mathrm{R}}^{-}(n)\right] P(n, t) \\
& +\left[\gamma_{\mathrm{L}}^{+}(n-1)+\gamma_{\mathrm{R}}^{+}(n-1)\right] P(n-1, t) \\
& +\left[\gamma_{\mathrm{L}}^{-}(n+1)+\gamma_{\mathrm{R}}^{-}(n+1)\right] P(n+1, t) .
\end{aligned}
$$

The rates and probabilities also determine the current. In the left junction the current is

$$
I_{\mathrm{L}}(t)=-e \sum_{n}\left[\gamma_{\mathrm{L}}^{+}(n)-\gamma_{\mathrm{L}}^{-}(n)\right] P(n, t) .
$$

In most cases we apply dc-voltages and are interested in the dc-current. In this case we need only the stationary solution of the master equationTand the currents in the left and right junctions are equal $I=I_{\mathrm{L}}=I_{\mathrm{R}}$.

As an example we consider a junction with symmetric bias $V_{\mathrm{L}}=-V_{\mathrm{R}}=$ $V / 2$. At low temperatures and low transport voltages (except at symmetry points) only two different charge states - and those transitions which connect both - have an appreciable probability. For instance $\Gamma$ if $n e<Q_{\mathrm{G}}<$ $(n+1) e$ we need to consider only $P(n)$ and $P(n+1)$ and the two transitions $\gamma_{\mathrm{L}}^{+}(n)$ and $\gamma_{\mathrm{R}}^{+}(n)$ increasing the island charge from $n$ to $n+1$ electrons $\Gamma$ as well as the two reverse transitions $\gamma_{\mathrm{L}}^{-}(n+1)$ and $\gamma_{\mathrm{R}}^{-}(n+1)$. The energy changes determining the rates $\gamma_{\mathrm{L}}^{+}(n)$ and $\gamma_{\mathrm{L}}^{-}(n+1)$ are

$$
\delta E_{\text {ch }}= \pm\left[\left(n+\frac{1}{2}-\frac{Q_{\mathrm{G}}}{e}\right) \frac{e^{2}}{C}-\frac{e V}{2}\right],
$$

respectively $\Gamma$ while for the transitions in the right junction $e V$ is replaced by $-e V$. In the 2-state limit the stationary probability and current become

$$
P(n)=\frac{\gamma_{\mathrm{L}}^{-}(n+1)+\gamma_{\mathrm{R}}^{-}(n+1)}{\gamma_{\Sigma}} ; P(n+1)=1-P(n)
$$




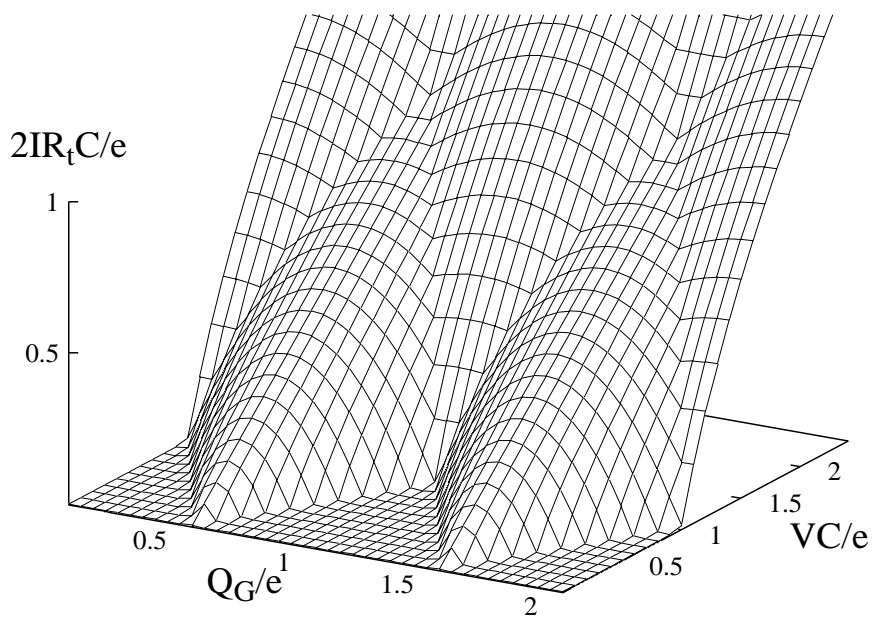

Figure 20. The current of a symmetric transistor is shown as a function of gate and transport voltage. At low temperatures and low transport voltages $V C / e<1$ only two charge states play a role $\Gamma$ and the Coulomb oscillations are clearly demonstrated. At larger transport voltages more charge states are involved.

$$
I=-e \frac{\gamma_{\mathrm{L}}^{+}(n) \gamma_{\mathrm{R}}^{-}(n+1)-\gamma_{\mathrm{R}}^{+}(n) \gamma_{\mathrm{L}}^{-}(n+1)}{\gamma_{\Sigma}},
$$

where $\gamma_{\Sigma}=\gamma_{\mathrm{L}}^{+}(n)+\gamma_{\mathrm{R}}^{+}(n)+\gamma_{\mathrm{L}}^{-}(n+1)+\gamma_{\mathrm{R}}^{-}(n+1)$.

This expression is readily analyzed by inspection of (29). At low temperatures the tunneling process in the left junction from $n$ to $n+1 \Gamma$ with rate $\gamma_{\mathrm{L}}^{+}(n)$ Iis allowed when $Q_{\mathrm{G}}-(n+1 / 2) e \geq-V C / 2$. On the other hand $\Gamma$ the transition which carries on the charge to the right electrode with rate $\gamma_{\mathrm{R}}^{-}(n+1)$ is allowed when $Q_{\mathrm{G}}-(n+1 / 2) e \leq V C / 2$. Both coexist in a window of width $C V$ around $Q_{\mathrm{G}}=(n+1 / 2) e$. The other two processes are not allowed simultaneously in fact they are suppressed in the window just mentioned. Therefore at low temperature the current is

$I=\frac{1}{4 R_{\mathrm{t}}}\left[V-\frac{4 e}{C^{2} V}\left(\frac{Q_{\mathrm{G}}}{e}-n-\frac{1}{2}\right)^{2}\right] \quad$ for $-\frac{V C}{2 e} \leq \frac{Q_{\mathrm{G}}}{e}-n-\frac{1}{2} \leq \frac{V C}{2 e}$,

while it vanishes outside the window. For simplicity we have assumed in (31) that the two junctions have the same tunneling resistance $R_{\mathrm{t}}=R_{\mathrm{t}, \mathrm{L}}=R_{\mathrm{t}, \mathrm{R}}$.

The current through a symmetric SET transistor is plotted as a function of transport and gate voltages in Fig. 20. For gate voltages such that $Q_{\mathrm{G}} / e$ is close to an integer $\Gamma$ the current vanishes below a threshold bias voltage $V_{\mathrm{th}}\left(Q_{\mathrm{G}}=n e\right)=e / C$. This is a manifestation of the Coulomb blockade. At non-integer values of $Q_{\mathrm{G}} / e$ the threshold voltage is lower $V_{\mathrm{th}}\left(Q_{\mathrm{G}}\right)=$ 


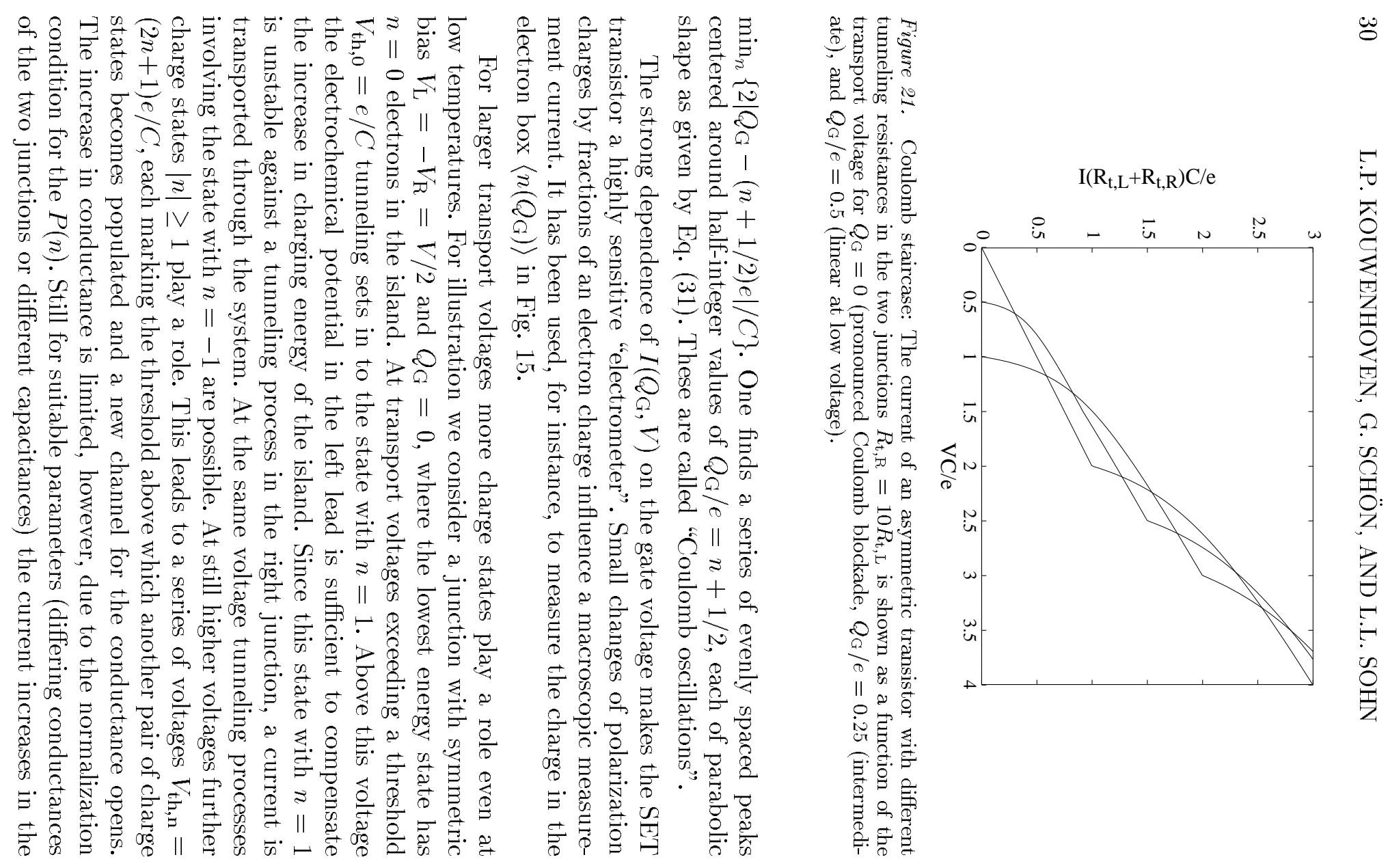


shape of a staircase. The phenomenon got accordingly the name "Coulomb staircase" [55]. The behavior is demonstrated in the plot of Fig. 21.

\subsection{HIGHER ORDER TUNNELING PROCESSES}

If sequential single-electron tunneling is suppressed by the Coulomb blockade Thigher-order processes such as coherent "cotunneling" through several junctions becomes crucial (Averin and Nazarov in Ref. [50]). As a specific example we consider a SET transistorTbiased such that the current in lowest-order perturbation theory vanishes (see Fig. 19). At low temperatures sequential tunneling is exponentially suppressed in this regime since the energy of a state with an excess charge on the island lies above the Fermi levels of the leads. On the other hand applied $\Gamma$ a higher-order tunneling process transferring an electron charge coherently through the total system is energetically allowed. In this case the state with an excess electron charge in the island exists only virtually. Standard second-order (or fourth Tdepending on the counting) perturbation theory yields the rate

$$
\gamma_{\mathrm{i} \rightarrow \mathrm{f}}=\frac{2 \pi}{\hbar}\left|\sum_{\psi} \frac{\left\langle i\left|H_{\mathrm{t}}\right| \psi\right\rangle\left\langle\psi\left|H_{\mathrm{t}}\right| f\right\rangle}{E_{\psi}-E_{\mathrm{I}}}\right|^{2} \delta\left(E_{\mathrm{i}}-E_{\mathrm{f}}\right)
$$

The energy of the intermediate virtual state lies above the initial one $\Gamma$ $E_{\psi}-E_{\mathbf{i}}>0$ C but it enters only into the denominator rather than into the exponent of the sequential tunneling rate. Hence the higher-order rate is nonzero even at very low temperatures.

When analyzing the process we have to pay attention to the following: (i) There are actually two channels which add coherently. Either an electron tunnels first from the left lead onto the island Tand then an electron tunnels from the island to the other lead. In this case the increase in charging energy of the intermediate state compared with the initial one is $\delta E_{\mathrm{L}}=$ $E_{\mathrm{ch}}\left(n+1, Q_{\mathrm{G}}\right)-E_{\mathrm{ch}}\left(n, Q_{\mathrm{G}}\right)-e V_{\mathrm{L}}$. Or an electron tunnels first out of the island to the right lead $\Gamma$ and another electron from the left lead replaces the charge. In this case the increase in energy of the intermediate state is $\delta E_{\mathrm{R}}=E_{\mathrm{ch}}\left(n-1, Q_{\mathrm{G}}\right)+e V_{\mathrm{R}}-E_{\mathrm{ch}}\left(n, Q_{\mathrm{G}}\right)$. Both amplitudes have to be added before the matrix element is squared.

(ii) The leads contain a macroscopic number of electrons. Therefore $\Gamma$ with overwhelming probability the outgoing electron will come from a different state than the one which the incoming electron occupies. Hence $\mathrm{Tafter}$ the process an electron-hole excitation is left in the island $\Gamma$ which explains why it is called "inelastic" cotunneling. This scenario is visualized in Fig. 22. 


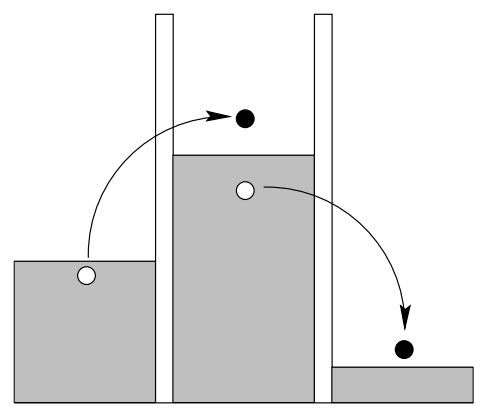

Figure 22. In an inelastic cotumneling process two electrons tunnel coherently $\Gamma$ i.e. in a single qutnum process $i$ in the left and right junction. The result is the transfer of charge through the system even in the Coulomb blockade regime. A particle-hole excitation is left in the island.

Transitions involving different excitations are added incoherently. The resulting rate for inelastic cotunneling is

$$
\begin{gathered}
\gamma_{\mathrm{cot}}=\frac{\hbar}{2 \pi \epsilon^{4} R_{\mathrm{t}, \mathrm{L}} R_{\mathrm{t}, \mathrm{R}}} \\
\times \int_{k \in \mathrm{L}} \mathrm{d} \epsilon_{k} \int_{q \in \mathrm{I}} \mathrm{d} \epsilon_{q} \int_{q^{\prime} \in \mathrm{I}} \mathrm{d} \epsilon_{q^{\prime}} \int_{k^{\prime} \in \mathrm{R}} \mathrm{d} \epsilon_{k^{\prime}} f\left(\epsilon_{k}\right)\left[1-f\left(\epsilon_{q}\right)\right] f\left(\epsilon_{q^{\prime}}\right)\left[1-f\left(\epsilon_{k^{\prime}}\right)\right] \\
\times\left[\frac{1}{\epsilon_{q}+\delta E_{\mathrm{L}}-\epsilon_{k}}+\frac{1}{\epsilon_{k^{\prime}}+\delta E_{\mathrm{R}}-\epsilon_{q^{\prime}}}\right]^{2} \delta\left(e V+\epsilon_{k}-\epsilon_{q}+\epsilon_{q^{\prime}}-\epsilon_{k^{\prime}}\right)
\end{gathered}
$$

At $T=0$ the integrals can be performed analytically. The result for in the Coulomb blockade regime $\left(e V \ll \delta E_{\mathrm{L}}, \delta E_{\mathrm{R}}\right)$ is

$$
\gamma_{\cot }=\frac{\hbar}{12 \pi e R_{\mathrm{t}, \mathrm{L}} R_{\mathrm{t}, \mathrm{R}}}\left(\frac{1}{\delta E_{\mathrm{L}}}+\frac{1}{\delta E_{\mathrm{R}}}\right)^{2} V^{3} .
$$

At finite temperatures forward and backward processes occur. They obey a detailed balance relation $\gamma_{\cot }(-V)=\exp \left(-e V / k_{\mathrm{B}} T\right) \gamma_{\cot }(V)$. The current then is

$$
I(V) \approx \frac{\hbar}{12 \pi e^{2} R_{\mathrm{t}, \mathrm{L}} R_{\mathrm{t}, \mathrm{R}}}\left(\frac{1}{\delta E_{\mathrm{L}}}+\frac{1}{\delta E_{\mathrm{R}}}\right)^{2}\left[(e V)^{2}+\left(2 \pi k_{\mathrm{B}} T\right)^{2}\right] V .
$$

In the Coulomb blockade regime of a SET transistor the $V^{3}$ dependence of the cotunneling current has been observed. In systems with $\mathrm{N}$ junctions a corresponding $\mathrm{N}$-th order process (or $2 \mathrm{~N}$-th orderTdepending on the counting) leads to a current $I \propto V^{2 N-1}$. As an example we consider $\mathrm{N}=4$ junctions with $C=10^{-15} \mathrm{~F}$ and tunneling resistance $R_{\mathrm{t}}$. In this 
case (see D. Estève in Ref. [50]) $\gamma_{\mathrm{cot}}=\left(2.5 \times 10^{-3} / \mathrm{sec}\right)(V / \mu \mathrm{V})^{7}\left(\mathrm{k} \Omega / R_{\mathrm{t}}\right)^{4}$. These cotunneling processes limit the accuracy of the single-electron turn-

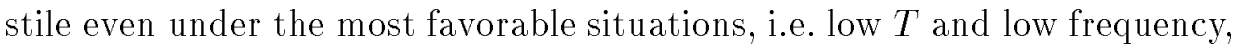
where thermally activated multi-electron transfer processes and missed cycles play little role.

The expression for the cotunneling rate presented above displays several important properties: (i) The expansion parameter is the dimensionless tunneling conductance $R_{\mathrm{K}} / R_{\mathrm{t}}$ Twhere the quantum resistance $R_{\mathrm{K}}$ serves as reference. (ii) The approximate expression given diverges when the intermediate and initial or final states are degenerate. This divergence is removed by life-time broadening effects. The complete cotunneling theory recently analyzed in Ref. [56] Гdescribes well the logarithmic temperature dependence observed in th experiments of the Saclay group on junctions with tunneling resistances comparable to the quantum resistance [57]. (iii) Higher order processes and eventually resonant tunneling processes are most essential near the points of degeneracy of the charging energy $Q_{\mathrm{G}} / e=n+1 / 2$. The Chapter of Schoeller in this volume presents the theoretical framework to describe tunneling beyond perturbation theory.

There exists also the process of "elastic cotunneling" where one electron tunnels through the total system leaving no excitations in the island. It is the dominant process in the Coulomb blockade regime of tunneling through a single-level quantum dot. However $i n$ the metallic junction it is usually not important $\Gamma$ since its rate is smaller by a prefactor $\propto 1 / \Omega_{\mathrm{I}} N_{\mathrm{I}}(0)$ (i.e. inversely proportional to the number of states of the island) compared to the inelastic cotunneling rate. The exception is the range of very low voltages and temperatures $k_{\mathrm{B}} T, e V \ll \sqrt{E_{\mathrm{C}} / \Omega N(0)}$ Tsince elastic cotunneling yields a current which is linear in the applied voltage.

Single-electron tunneling is also influenced by the response and the fluctuations of the electromagnetic circuit where it is embedded. This is particularly important in single junctions. In this case Coulomb blockade effects can only be observed if the junction is in series with a nearby large resistor of the order of the quantum resistance or larger. In systems of junctions the tunneling resistance of one junction usually provides the required decoupling of the remaining junctions and single-electron effects are observable. Here we do not have the space to present this theory. It is reviewed in the article by Ingold and Nazarov in Ref. [50].

\subsection{SINGLE-ELECTRON TUNNELING THROUGH QUANTUM DOTS}

In this Subsection we describe the influence of Coulomb blockade phenomena on single-electron tunneling through ultrasmall quantum dots. The important difference compared to the metallic case is the quantization of 
the energy levels inside the quantum dot with typical separation $\delta$. It can be resolved in transport experiments $\Gamma$ when the level spacing exceeds the temperature $\delta>k_{\mathrm{B}} T$. In this case one encounters the phenomenon of resonant tunneling through discrete levels.

Many of the experiments showing single-electron effects in quantum dots can be explained by lowest order perturbation theory in tunneling. As in the metallic case The theory is based on a classical master equation with golden-rule rates which describe incoherent transport through the device. This means that the electrons tunnel sequentiallyГi.e. they loose their phase memory before the next tunneling process occurs. The "orthodox theory" $\Gamma$ initially developed for metallic islands [45Г 46$] \Gamma$ was later generalized to quantum dots with discrete spectra [58Г59Г60Г61]. In this Section we describe this golden-rule theory for the simplest systems. Quantum dots with exact many-body wave functions in the few electron limit have been studied in Ref. [62Г $63 \Gamma 64] \Gamma$ while coupled quantum dots have been considered in Ref. [65Г66]. The effect of time-dependent fields have been described in Refs [67Г68] for metallic junctions and in Ref. [69] for the Coulomb blockade model and in Refs. $[70 \Gamma 71 \Gamma 72]$ for the metallic case in the presence of a heat bath. Various extensions $\Gamma$ incl. experimental results and further references $\Gamma$ will be reviewed in the Chapter of Kouwenhoven $e t$ al. later in this volume. A powerful theory which allows a consistent treatment of higher order tunneling processes and further generalizations will be presented in the Chapter by Schoeller.

The electron tunneling through a quantum dot is described by the Hamiltonian $H=H_{\text {res }}+H_{\mathrm{D}}+H_{\mathrm{t}}$ Twhere

$$
\begin{aligned}
H_{\mathrm{res}} & =\sum_{\mathrm{r}=\mathrm{L}, \mathrm{R}}\left[\sum_{k, \sigma} \epsilon_{k \mathrm{r}} a_{k, \sigma \mathrm{r}}^{\dagger} a_{k, \sigma \mathrm{r}}+e V_{\mathrm{r}} \hat{n}_{\mathrm{r}}\right], \\
H_{\mathrm{D}} & =\sum_{\mathrm{s}} E_{\mathrm{s}}|s><s| \\
H_{\mathrm{t}} & =\sum_{\mathrm{r}=\mathrm{L}, \mathrm{R}} \sum_{k, l, \sigma} T_{k l}^{\mathrm{r}} a_{k, \sigma \mathrm{r}}^{\dagger} a_{l, \sigma \mathrm{D}}+\text { h.c. }
\end{aligned}
$$

describe the reservoirs $\Gamma$ the $\operatorname{dot}$ Tand the tunneling $\Gamma$ respectively.

The reservoirs are assumed to have noninteracting single-particle states labeled by the reservoir index r $\Gamma$ wave vector $k$ and spin $\sigma$. The voltage of reservoir $\mathrm{r}$ is denoted by $V_{\mathrm{r}}$ Tand $\hat{n}_{\mathrm{r}}$ denotes the total particle number.

The eigenstates of the isolated dot are denoted by $|s\rangle$ with energy $E_{\mathrm{s}}$. For the Coulomb blockade model $\Gamma$ the states $\mid s>$ of the dot are specified by the set of all occupation numbers for the single particle states: $|s\rangle=$ 
$\mid\left\{n_{l, \sigma \mathrm{D}}\right\}>$. In this case $\Gamma$ the dot energy is given by

$$
E_{\mathrm{s}}=\sum_{l, \sigma} \epsilon_{l \mathrm{D}} \hat{n}_{l, \sigma \mathrm{D}}+E_{\mathrm{C}}\left(n-n_{\mathrm{G}}\right)^{2},
$$

where $\hat{n}$ is the particle number operator of the dot. The charging energy $E_{\mathrm{C}}=e^{2} / 2 C$ and the gate charge $Q_{\mathrm{G}}=-e n_{\mathrm{G}}=\sum_{\mathrm{i}=\mathrm{L}, \mathrm{R}, \mathrm{G}} C_{\mathrm{i}} V_{\mathrm{i}}$ coincide with the expressions (13) and (15) introduced in the metallic case. The general notation in terms of the many-body wave functions $\mid s>$ is introduced here to include cases where the states of the dot cannot be described by single particle states $\Gamma$ see e.g. Refs. [73Г $62 \Gamma 63 \Gamma 74]$.

The tunneling part describes charge transfer processes between the reservoirs and the dot. The tunneling matrix elements are conveniently combined in the spectral function

$$
\Gamma_{\mathrm{r}, l l^{\prime}}(E)=\frac{2 \pi}{\hbar} \sum_{k} T_{k l}^{\mathrm{r}^{*}} T_{k l^{\prime}}^{\mathrm{r}} \delta\left(E-\epsilon_{k \mathrm{r}}\right),
$$

which depends on the energy and the single-particle states $l, l^{\prime}$ involved. A typical value of the spectral function defines an energy scale $\Gamma$ which characterizes the intrinsic broadening of the single-particle states of the dot by quantum fluctuations from tunneling. If $\Gamma \ll k_{\mathrm{B}} T \Gamma$ thermal fluctuations dominate over quantum fluctuations $\Gamma$ and we can use golden-rule theory.

The reservoirs are treated as large systems in equilibrium described by the grand canonical density matrix $\rho_{\text {res }}^{\text {eq }}$. The tunneling rate for a transition of the dot from state $\left|s^{\prime}\right\rangle$ to $|s\rangle$ when $p= \pm 1$ charges have been added from reservoir $r$ to the dot is given by the golden-rule expression

$$
\gamma_{\mathrm{r}, s s^{\prime}}^{p}=\frac{2 \pi}{\hbar} \sum_{\substack{\chi \chi^{\prime} \\ n_{\mathrm{r}}\left(\chi^{\prime}\right)=n_{\mathrm{r}}(\chi)+p}} \rho_{\mathrm{res}}^{\mathrm{eq}}\left(\chi^{\prime}\right)|<\chi s| H_{\mathrm{t}}\left|\chi^{\prime} s^{\prime}>\right|^{2} \delta\left(E_{\mathrm{s}}-E_{\mathrm{s}^{\prime}}+E_{\chi}-E_{\chi^{\prime}}-p \mu_{\mathrm{r}}\right) .
$$

Here $\chi$ denote the states of the reservoirs with particle numbers $n_{\mathrm{r}}(\chi)$. The energy conserving $\delta$-function includes the change $\mu_{\mathrm{r}}=e V_{\mathrm{r}}$ of the electrostatic energy which is regarded here as the effective electrochemical potential of reservoir $r$. The change of the electrostatic energy of the dot is included in $E_{s}-E_{s^{\prime}}$.

Inserting the tunneling Hamiltonian $H_{\mathrm{t}}$ from $\mathrm{Eq}$. (38) into the goldenrule rate (41) yields

$$
\gamma_{\mathrm{r}, s s^{\prime}}^{p}=\sum_{l l^{\prime}} \Gamma_{\mathrm{r}, l l^{\prime}}\left(E_{s}-E_{s^{\prime}}-\mu_{\mathrm{r}}\right)<s\left|a_{l \mathrm{D}}^{\dagger}\right| s^{\prime}><s^{\prime}\left|a_{l^{\prime} D}\right| s>f_{\mathrm{r}}^{p}\left(E_{s}-E_{s^{\prime}}\right),
$$

where $f_{\mathrm{r}}^{+}(\omega)=f\left(\omega-\mu_{\mathrm{r}}\right)$ is the Fermi distribution of reservoir $\mathrm{r} \Gamma$ while $f_{\mathrm{r}}^{-}=1-f_{\mathrm{r}}^{+}$ 
The rates can be used as an input for a master equation. Consequently the stationary dc-probability distribution $P_{\mathrm{s}}$ for the dot and the stationary dc-tunneling current in reservoir $\mathrm{r}$ can be calculated from

$$
\begin{aligned}
0 & =\sum_{s^{\prime}}\left[\gamma_{s s^{\prime}} P\left(s^{\prime}\right)-\gamma_{s^{\prime} s} P(s)\right], \\
I_{\mathrm{r}} & =e \sum_{s s^{\prime}}\left[\gamma_{\mathrm{r}, s s^{\prime}}^{+} P\left(s^{\prime}\right)-\gamma_{\mathrm{r}, s^{\prime} s}^{-} P(s)\right],
\end{aligned}
$$

with $\gamma_{s s^{\prime}}=\sum_{\mathrm{r} p} \gamma_{\mathrm{r}, s s^{\prime}}^{p}$. Current conservation $\sum_{\mathrm{r}} I_{\mathrm{r}}=0$ follows from the property

$$
\sum_{\mathrm{r}}\left[\gamma_{\mathrm{r}, s s^{\prime}}^{+}-\gamma_{\mathrm{r}, s s^{\prime}}^{-}\right]=\gamma_{s s^{\prime}}\left(n_{s}-n_{s^{\prime}}\right)
$$

where $n_{s}$ denotes the number of particles in the dot in state $s$.

The rates satisfy the detailed balance relation $\gamma_{\mathrm{r}, s^{\prime} s}^{-} / \gamma_{\mathrm{r}, s s^{\prime}}^{+}=\exp \left[\beta\left(E_{s}-\right.\right.$ $\left.\left.E_{s^{\prime}}-\mu_{\mathrm{r}}\right)\right]$. As a consequence the equilibrium solution of the master equation (43) is the grand canonical distribution

$$
P^{\mathrm{eq}}(s)=\frac{1}{Z} e^{-\beta\left(E_{s}-\mu n_{s}\right)},
$$

which applies when all electrochemical potentials are the same $\mu_{\mathrm{r}}=\mu$. In this case the dc-current (44) is zero.

Using detailed balance we can write the tunneling rates as

$$
\gamma_{\mathrm{r}, s s^{\prime}}^{+}=f_{\mathrm{r}}^{+}\left(E_{s}-E_{s^{\prime}}\right) \gamma_{\mathrm{r}, s s^{\prime}}^{\Sigma} \quad, \quad \gamma_{\mathrm{r}, s^{\prime} s}^{-}=f_{\mathrm{r}}^{-}\left(E_{s}-E_{s^{\prime}}\right) \gamma_{\mathrm{r}, s s^{\prime}}^{\Sigma},
$$

where

$$
\gamma_{\mathrm{r}, s s^{\prime}}^{\Sigma}=\gamma_{\mathrm{r}, s s^{\prime}}^{+}+\gamma_{\mathrm{r}, s^{\prime} s}^{-}
$$

is the sum of tunneling 'in' and tunneling 'out' rates. As a consequence the current (44) can be expressed as

$$
I_{\mathrm{r}}=e \sum_{s s^{\prime}} \gamma_{\mathrm{r}, s s^{\prime}}^{\Sigma}\left[f_{\mathrm{r}}^{+}\left(E_{s}-E_{s^{\prime}}\right) P\left(s^{\prime}\right)-f_{\mathrm{r}}^{-}\left(E_{s}-E_{s^{\prime}}\right) P(s)\right] .
$$

A current can flow through the structure if both the tunneling 'in' and tunneling 'out' rate are non-zero. At low temperatures we consider the transition between two dot states $s_{n} \leftrightarrow s_{n+1} \Gamma$ with $n$ and $n+1$ particles in the dot Trespectively. For tunneling 'in' from reservoir $\mathrm{r}$ we need $\Delta E=$ $E_{s_{n+1}}-E_{s_{n}}<\mu_{\mathrm{r}}$ Tand a similar relation for tunneling 'out' to reservoir r'. Both conditions are satisfied simultaneously if the excitation energy $\Delta E$ lies in the window of the effective potentials of the reservoirs $\Gamma \mu_{\mathrm{r}^{\prime}}<\Delta E<\mu_{\mathrm{r}}$.

The energy- and state-dependence of the spectral function $\Gamma_{\mathrm{r}, l l^{\prime}}(\omega)$ accounts for the energy-dependence of the density of states in the reservoirs $\Gamma$ 
and mesoscopic fluctuations of the conductance peak heights. Here we concentrate on the simplest case and set

$$
\Gamma_{\mathrm{r}, l l^{\prime}}(\omega) \approx \delta_{l l^{\prime}} \Gamma_{\mathrm{r}}
$$

In this case we find from (42) that the sum of the tunneling 'in' and 'out' rates defined in (48) Tdiffers from this scale

$$
\gamma_{\mathrm{r}, s s^{\prime}}^{\Sigma}=\Gamma_{\mathrm{r}} \sum_{l}|<s| a_{l \mathrm{D}}^{\dagger}\left|s^{\prime}>\right|^{2}
$$

only by a constant factor $\Gamma$ since the Fermi functions have canceled out. Inserting this relation in (49) Tand using current conservation $\sum_{\mathrm{r}} I_{\mathrm{r}}=0$ to eliminate the term which is independent of the Fermi functions $\Gamma$ we find

$$
\begin{aligned}
I_{\mathrm{r}}=e \sum_{\mathrm{r}^{\prime} s s^{\prime}} \frac{\Gamma_{\mathrm{r}} \Gamma_{\mathrm{r}^{\prime}}}{\Gamma} \sum_{l}|<s| a_{l \mathrm{D}}^{\dagger}\left|s^{\prime}>\right|^{2}\left[P(s)+P\left(s^{\prime}\right)\right] \\
\quad \times\left[f_{\mathrm{r}}\left(E_{s}-E_{s^{\prime}}\right)-f_{\mathrm{r}^{\prime}}\left(E_{s}-E_{s^{\prime}}\right)\right],
\end{aligned}
$$

with $\Gamma=\sum_{\mathrm{r}} \Gamma_{\mathrm{r}}$. This formula is frequently used in the literature. In linear response $\Gamma$ it reduces to a form first derived by Beenakker [59]. In this case we set $\mu_{\mathrm{r}}=\mu+e \delta V_{\mathrm{r}}$ Tand find

$$
I_{\mathrm{r}}=\sum_{\mathrm{r}^{\prime}} G_{\mathrm{r} r^{\prime}}\left(\delta V_{\mathrm{r}}-\delta V_{\mathrm{r}^{\prime}}\right)
$$

where the conductance is

$G_{\mathrm{rr}^{\prime}}=-e^{2} \sum_{s s^{\prime}} \frac{\Gamma_{\mathrm{r}} \Gamma_{\mathrm{r}^{\prime}}}{\Gamma} \sum_{l}\left|\left\langle s\left|a_{l \mathrm{D}}^{\dagger}\right| s^{\prime}\right\rangle\right|^{2}\left[P(s)^{\mathrm{eq}}+P\left(s^{\prime}\right)^{\mathrm{eq}}\right] f^{\prime}\left(E_{s}-E_{s^{\prime}}-\mu\right)$.

In the general case $\Gamma$ many excitation energies $E_{s}-E_{s^{\prime}}$ can lie between $\mu_{\mathrm{r}}$ and $\mu_{\mathrm{r}^{\prime}}$ and are relevant for transport. However $\Gamma$ only those transitions $s^{\prime} \rightarrow s$ will occur for which the initial probability $P\left(s^{\prime}\right)$ is not too small. For temperatures and bias voltages smaller than the level spacing $\delta$ and the charging energy $E_{\mathrm{ch}}$ Tonly the ground states $s_{n}^{0}$ of the dot will have nonzero occupation probability. This means that only the energies

$$
\Delta_{n}=E_{s_{n+1}^{0}}-E_{s_{n}^{0}}
$$

are relevant. The transition from $s_{n}^{0}$ to an excited state $s_{n+1}^{*}$ does not occur since $\Gamma$ upon increasing the gate voltage $\Gamma$ the transition $s_{n}^{0} \rightarrow s_{n+1}^{0}$ occurs sooner and afterwards the dot is already in the $n+1$-particle ground state. Thus $\Gamma$ we obtain the same physical picture as shown in Fig. 19 with the 


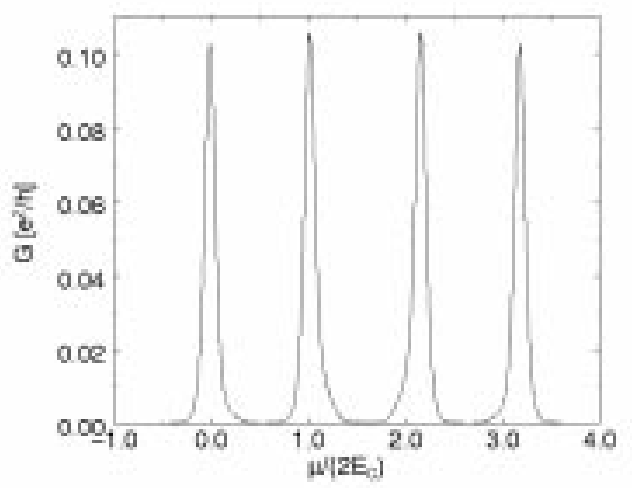

Figure 23. Linear conductance versus $\mu$ for two doubly degenerate levels with $\bar{\epsilon}_{1}=0 \Gamma$ $\bar{\epsilon}_{2}=25 \Gamma \Gamma T=5 \Gamma \Gamma E_{\mathrm{C}}=75 \Gamma \Gamma$ and $\Gamma^{\mathrm{L}}=\Gamma^{\mathrm{R}}=\Gamma / 2$. The distance between the second and third main resonance is larger due to the finite level spacing. All resonances involving excited states are hidden.

only difference that the distance $\Delta$ between adjacent excitation energies is no longer a constant.

This behavior is reflected in the formula (54) for the conductance matrix in linear response. Due to the derivative of the Fermi function $\Gamma$ the conductance will be maximal when $\mu$ coincides with one of the excitation energies within a range set by the temperature. The energy difference $E_{s}-E_{s^{\prime}}$ is varied experimentally by the gate voltage. Thus $\mathrm{T}$ the conductance shows a series of resonances with varying distance between the peaks and a line shape which is approximately given by the derivative of the Fermi distribution function. Between the resonancesTtransport is not possible and the system is in the Coulomb blockade regime. As an example $\Gamma$ we show the Coulomb oscillations in Fig. 23 for the Coulomb blockade model with two spin-degenerate levels with energies $\epsilon_{1}<\epsilon_{2}$. According to (39) the excitation energies $\Gamma$ describing the energy changes of the dot when a particle is added in level $l$ to a state with $n$ particles Tare given by

$$
\Delta_{n l}=\bar{\epsilon}_{l \mathrm{D}}+2 n E_{\mathrm{C}}
$$

Here $\bar{\epsilon}_{l \mathrm{D}}=\epsilon_{l \mathrm{D}}+E_{\mathrm{C}}\left(1-n_{\mathrm{G}}\right)$ describes the effective level position $\Gamma$ which is tuned by the gate voltage. As a consequence $\Gamma$ we observe four resonances corresponding to the excitation energies $\Delta_{01}=\bar{\epsilon}_{1} \Gamma \Delta_{11}=\bar{\epsilon}_{1}+2 E_{\mathrm{C}} \Gamma \Delta_{22}=$ $\bar{\epsilon}_{2}+4 E_{\mathrm{C}} \Gamma$ and $\Delta_{32}=\bar{\epsilon}_{2}+6 E_{\mathrm{C}}$. As explained above $\Gamma$ all other excitation energies $\Delta_{02}=\bar{\epsilon}_{2} \Gamma \Delta_{12}=\bar{\epsilon}_{2}+2 E_{\mathrm{C}} \Gamma \Delta_{21}=\bar{\epsilon}_{1}+4 E_{\mathrm{C}} \Gamma$ and $\Delta_{31}=\bar{\epsilon}_{1}+6 E_{\mathrm{C}}$ are hidden because they involve excited states. 


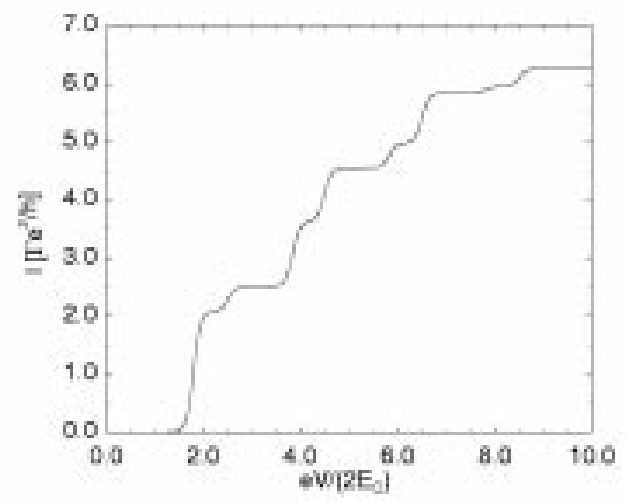

Figure 24. The dc current in nonlinear response versus $e V=e\left(V_{\mathrm{L}}-V_{\mathrm{R}}\right) \Gamma$ with $V_{\mathrm{L}}=-V_{\mathrm{R}}=V / 2$ and $C_{\mathrm{G}} V_{\mathrm{G}} / e=1$ fixed $\Gamma$ for two doubly degenerate levels with $\bar{\epsilon}_{1}=0 \Gamma$ $\bar{\epsilon}_{2}=50 \Gamma$. The other parameters are $T=5 \Gamma \Gamma E_{\mathrm{C}}=75 \Gamma \Gamma$ and $\Gamma^{\mathrm{L}}=\Gamma^{\mathrm{R}}=\Gamma / 2$. All one-particle excitations of the dot are visible.

At finite bias voltage all excitations are in principle visible since the excited states acquire a finite occupation probability. This holds at least in the absence of certain selection rules arising from the matrix element $<s\left|a_{l \mathrm{D}}^{\dagger}\right| s^{\prime}>$ in (52). For a constant density of states of the leads the I-Vcharacteristic shows steps each time a new excitation becomes relevant. This result is shown in Fig. 24 for the same example as before. Equivalently the differential conductance $d I / d V$ shows peaks as function of the bias voltage. As can be seen $\Gamma$ all eight excitation energies mentioned before are visible.

The effects of strong correlations on the dot are not only reflected in the increase of the distance between adjacent resonances but also in the line shape of an individual peak as a function of the applied gate voltage. To show this explicitly $\mathrm{T}$ we consider the Coulomb blockade model for a single spin-degenerate state with energy $\epsilon$ on the dot. For $E_{\mathrm{C}} \gg T$ we can restrict ourselves to the transition between an empty and a singly occupied $\operatorname{dot} \Gamma n=0,1$. According to relation (56) $\Gamma$ the excitation energy for this transition is given by $\Delta=\bar{\epsilon}$. From the master equation and (52) we find

$$
\begin{aligned}
I_{\mathrm{r}} & =2 e \sum_{\mathrm{r}^{\prime}} \frac{\Gamma_{\mathrm{r}} \Gamma_{\mathrm{r}^{\prime}}}{\Gamma} \frac{1}{1+\sum_{\mathrm{r}^{\prime \prime}} \frac{\Gamma_{\mathrm{r}^{\prime \prime}}}{\Gamma} f_{\mathrm{r}^{\prime \prime}}(\Delta)}\left[f_{\mathrm{r}}(\Delta)-f_{\mathrm{r}^{\prime}}(\Delta)\right], \\
G_{\mathrm{rr}^{\prime}}(\mu) & =-2 e^{2} \frac{\Gamma_{\mathrm{r}} \Gamma_{\mathrm{r}^{\prime}}}{\Gamma} \frac{1}{1+f(\Delta-\mu)} f^{\prime}(\Delta-\mu) .
\end{aligned}
$$

The current contains an asymmetry factor $1 /\left(1+\sum_{\mathrm{r}} \frac{\Gamma_{\mathrm{r}}}{\Gamma} f_{\mathrm{r}}(\Delta)\right)$ which is absent either for a nondegenerate level or for the noninteracting case $E_{\mathrm{C}}=$ 
0 . This factor arises from correlations since double occupancy of the dot is forbidden. This gives rise to particle-hole asymmetry and $\Gamma$ consequently $\Gamma$ to an asymmetric line shape of the differential conductance as a function of $\Delta$ as shown in Fig. 25 for finite bias voltage. The maximal value of the conductance in linear response is given by

$$
G_{\mathrm{rr}^{\prime}}^{\max }=4 \pi \frac{e^{2}}{h} \frac{\Gamma_{\mathrm{r}} \Gamma_{\mathrm{r}^{\prime}}}{\Gamma} \frac{2}{3 T} .
$$

For a nondegenerate level or for the noninteracting case with one degenerate level $\Gamma$ the factor $2 / 3$ has to be replaced by $1 / 2$ or $1 \Gamma$ respectively. This can be easily understood. At the maximum of the conductance several states of the dot have the same probability. For large charging energy the doubly occupied state can be excluded $\Gamma$ and we have two degenerate excitations which can be used for transport $\Gamma$ and three possible states of the dot (the empty dot and two degenerate states with one electron). Each excitation contributes equally to the current but has to be multiplied with the probability $1 / 3$ of the initial state. This explains the factor $2 / 3$. For a nondegenerate level we have only one excitation and two states $\Gamma$ resulting in a factor $1 / 2$. For a noninteracting model with one degenerate level we have four excitations (two for each transition $n=0 \rightarrow n=1$ and $n=1 \rightarrow n=2$ ) and four possible statesTgiving a factor 1 . The reduction of the current by Coulomb repulsion is obvious Tsince certain processes are blocked. In contrast to the noninteracting case $\Gamma$ we have seen that the presence of degenerate states does not give rise to a pure multiplicative factor of the degeneracy. The reason is that Coulomb interaction induces a correlation between the levels. When one level is occupied Tthe other is not allowed to be occupied due to the strong on-site Coulomb repulsion.

Selection rules occur due to the matrix element $\left|\left\langle s\left|a_{l \mathrm{D}}^{\dagger}\right| s^{\prime}\right\rangle\right|^{2}$ in (52). Spin conservation allows only transitions where the total spin of the states $s$ and $s^{\prime}$ differs by $\pm 1 / 2$. For the discussion of spin blockade effects and related negative differential conductances we refer to Refs. [62Г63Г 74].

\section{Conclusions}

Many further fundamental concepts of mesoscopic electron transport could not be included in this IntroductionTbut will be covered in following Chapters. Interference and localization effects and extensions will be discussed in the Chapters of Stern and of Imry. The Chapter by Kouwen-

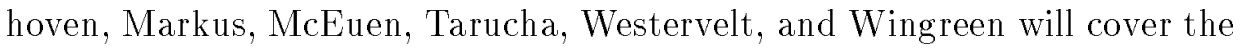
wide field of electron transport through quantum dots. Eaves includes in his Chapter concepts related to chaos. De Jong and Beenakker review noise properties of electron transport. Also Büttiker and Christen's Chapter deals 


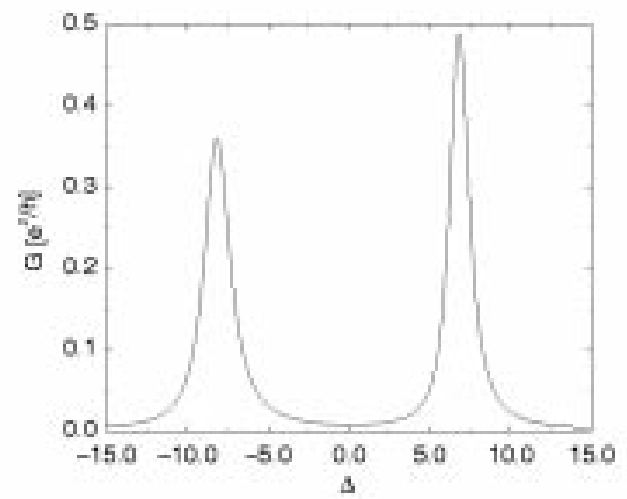

Figure 25. The differential conductance as a function of $\Delta=\bar{\epsilon}$ for a two-fold degenerate level with large charging energy $E_{\mathrm{C}}$ so that double occupancy can be neglected. $T=0.25 \Gamma \Gamma \mu_{\mathrm{L}}=-\mu_{\mathrm{R}}=15 \Gamma \Gamma$ and $\Gamma^{\mathrm{L}}=\Gamma^{\mathrm{R}}=\Gamma / 2$.

with extensions to time-dependent phenomena. A systematic discussion of tunneling beyond perturbation theory is presented in Schoeller's Chapter $\Gamma$ while Fisher and Glazman discuss transport in 1D interacting systems.

Superconductivity adds further degrees of freedom to mesoscopic electron transport. The properties of normal metal-superconductor heterostructures are described in the Chapter of the Saclay group $\Gamma$ while Fazio and Schön describe single-charge tunneling in superconducting junction systems and further review the theory of quantum transport in NS heterostructures. The very existence of superconductivity in ultrasmall particles is investigated in the article of Ralph et al. Twhile van Wees and Takayanagi address transport through semiconductor - superconductor systems.

The field of scanning probe microscopy has advanced substantially in recent years. It is reviewed by Sohn et al. The transport through quantum point contacts still reveals new results as described in two Chapters by van Ruitenbeek and by Garcia et al.

We included in this Book also two peripheral Chapters. Yamamoto describes concepts of quantum optics to solid state physicists $\Gamma$ while DiVincenzo reviews the novel field of quantum computing.

\section{References}

1. Molecular Beam Epitaxy: Fundamentals and Current StatusTM. A. Herman and H. SitterT Springer-VerlagГNY (1985); The Technology and Physics of Molecular Beam EpitaxyГed. E. H. C. ParkerГPlenum Press TNY (1985)

2. H. van HoutenГB. J. van WeesГM. G. J. HeijmanTand J. P. AndreTAppl. Phys. Lett. $49 \Gamma 1781(1986)$. 
3. T. J. ThorntonГM. PepperГH. AhmedГD. AndrewsTand G. J. DaviesTPhys. Rev. Lett. 56Г1198 (1986).

4. H. Z. ZhengГH. P. WeiГD. C. TsuiTand G. WeimannTPhys. Rev. B 34Г5635 (1986).

5. S. E. LauxГD. J. FrankГand F. SternГSurf. Sci. 196Г101 (1988).

6. H. Ehrenreich and D. TurnbullTeds. T Solid State Physics (Academic Press N New YorkT1991).

7. Yu. V. SharvinT Zh. Eksp. Teor. Fiz. 48Г 984 (1965) [Sov. Phys. JETP 21Г 655 (1965)].

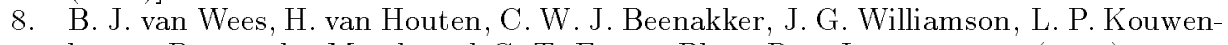

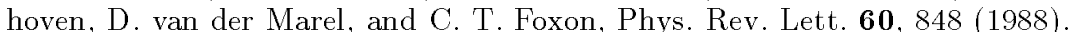

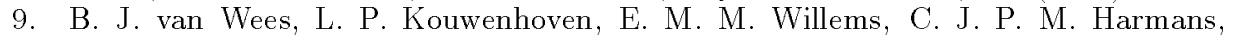

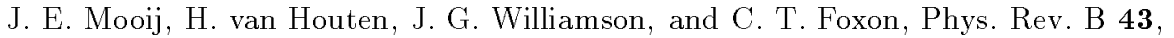
12431 (1991).

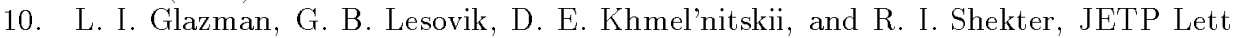

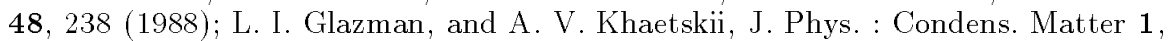
5005 (1989).

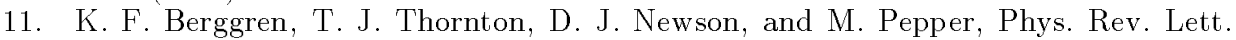
57Г1769 (1986).

12. R. LandauerГ IBM J. Res. Dev. 1Г 223 (1957); Phys. Lett. 85АГ 91 (1981); J. Phys. Condens. Matter 1Г8099 (1989).

13. A. Szafer and A. D. StoneГ Phys. Rev. Lett. 62Г300 (1989); E. G. Haanappel and D. van der MarelГPhys. Rev. B 39Г5484 (1989); G. Kirczenow $\Gamma$ Phys. Rev. В 39Г 10452 (1989).

14. Several authors have obtained transmission resonances in the calculated conductance of narrow constrictions $\Gamma$ see Ref. [13].

15. J. Nixon and J. Davies P Phys. Rev. B 41Г7929 (1990).

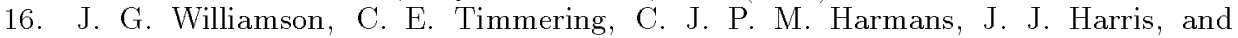
C. T. FoxonГPhys. Rev. B 42Г7675 (1990).

17. B. I. Halperin Phys. Rev. B 25Г2185 (1982).

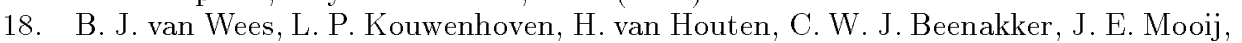
C. T. FoxonTand J. J. HarrisTPhys. Rev. B 38Г3625 (1988).

19. L. P. KouwenhovenTB. J. van WeesГC. J. P. M. HarmansГJ. G. WilliamsonГH. van

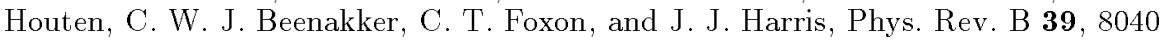
(1989).

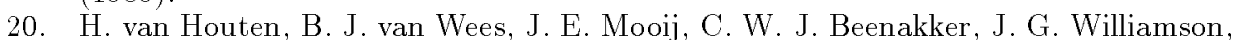
and C. T. FoxonTEurophys. Lett. 5T721 (1988); C. W. J. BeenakkerГH. van Houten and B. J. van WeesГEurophys. Lett. 7Г359 (1988); H. van Houten et al.TPhys. Rev. B 39Г8556 (1989).

21. M. BüttikerГPhys. Rev. Lett. 57Г1761Г(1986).

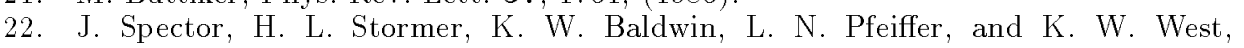
Surf. Sci. 228Г283 (1990).

23. P. StredaГJ. KuceraГand A. H. MacDonaldГPhys. Rev. Lett. 59Г1973 (1987).

24. J. K. Jain and S. A. KivelsonTPhys. Rev. Lett. 60Г1542 (1988).

25. M. BüttikerГPhys. Rev. B 38Г9375 (1988).

26. M. Reed Гed. TSemiconductors and Semimetals (Academic Press $\Gamma$ New York $\Gamma 1990$ ).

27. B. J. van WeesTE. M. M. WillemsTC. J. P. M. HarmansTC. W. J. BeenakkerГH. van

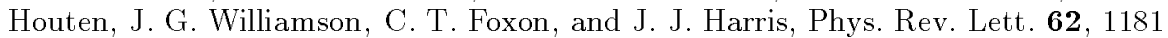
(1989).

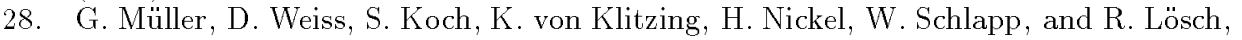
Phys. Rev. B 42Г7633 (1990).

29. S. KomiyamaГH. HiraiГS. SasaГand S. HiyamizuГPhys. Rev. B 40Г12566 (1989).

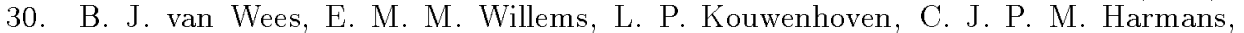
J. G. WilliamsonTC. T. FoxonTand J. J. HarrisTPhys. Rev. B 39Г8066 (1989).

31. B. W. AlphenaarTP. L. McEuenTR. G. WheelerTand R. N. Sacks PPhys. Rev. Lett. $64 \Gamma 677$ (1990). 


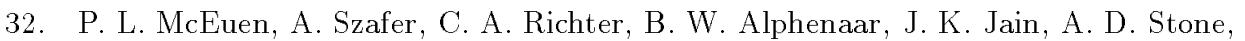
R. G. WheelerTand R. N. SacksГPhys. Rev. Lett. 64Г2062 (1990).

33. For a review see T. Chakraborty and P. Pietiläinen T The fractional quantum Hall effect TSpringer series in Solid-State Sciences 85 (Springer-Verlag 1988).

34. For a review see S. C. ZhangTInt. J. of Mod. Phys. ВГ 6Г25 (1992).

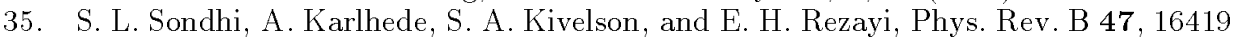

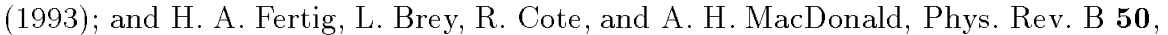
11018 (1994).

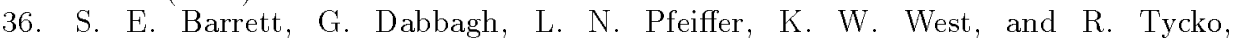
Phys. Rev. Lett. 74Г5112 (1995).

37. L. P. KouwenhovenTunpublished.

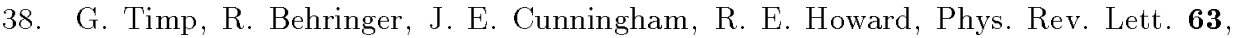
2268 (1989).

39. L. P. KouwenhovenT B. J. van Wees N. C. van der VaartTC. J. P. M. Harmans C. E. Timmering Tand C. T. FoxonTPhys. Rev. Lett $64 \Gamma 685$ (1990).

40. A. M. Chang and J. E. Cunningham TPhys. Rev. Lett. 69Г2114 (1992).

41. C. W. J. BeenakkerГPhys. Rev. Lett. 64Г216 (1990).

42. A. H. MacDonald Phys. Rev. Lett. 64Г220 (1990).

43. A. M. Chang $\Gamma$ Solid State Commun. $74 \Gamma 871$ (1990).

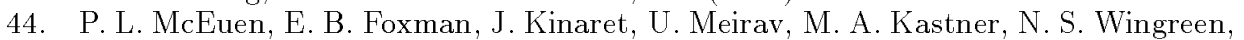
and S. J. Wind Phys. Rev B $45 \Gamma 11419$ (1992); D. B. ChklovskiiГ B. I. Shklovskii and L. I. GlazmanTPhys. Rev. B 46Г4026 (1992).

45. I. O. Kulik and R. I. Shekhter $\Gamma$ Zh. Eksp. Teor. Fiz. 68Г 623 (1975) [Sov. Phys. JETP 41Г308 (1975)].

46. D. V. Averin and K. K. LikharevГ J. Low Temp. Phys. 62Г 345 (1986); and in Mesoscopic Phenomena in SolidsTB. L. AltshulerTP. A. Lee and R. A. WebbTeds. $\Gamma$ p. 173 (Elsevier $\Gamma$ Amsterdam $\Gamma 1991$ ).

47. T. A. Fulton and G. J. DolanTPhys. Rev. Lett. 59Г109 (1987).

48. L. J. Geerligs et al. ГPhys. Rev. Lett. 64Г2691 (1990).

49. H. Pothier et al. ГPhysica B 169Г573 (1991)ГEurophys. Lett. 17Г249 (1992)

50. Single Charge Tunneling $\Gamma$ NATO ASI Series $\Gamma$ Vol. B $294 \Gamma$ eds. H. Grabert and M. H. Devoret $\Gamma$ (New York $\Gamma$ Plenum Press 1992).

51. Mesoscopic Superconductivity $\Gamma$ Proceedings of the NATO ARW edited by F. W. J. Hekking ГG. SchönTand D. V. Averin Physica B 203 (1994).

52. G. Schön and A. D. ZaikinT Phys. Rep. 198Г237 (1990).

53. L. I. Glazman and K. A. MatveevTSov. Phys. JETP. 71 1031 (1990); K. A. MatveevT Sov. Phys. JETP 72Г892 (1991).

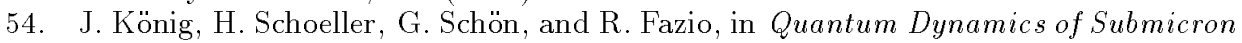

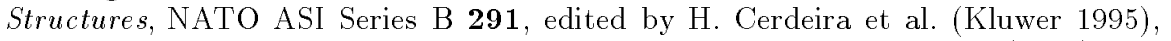
p. 221; J. König ГH. SchoellerГ and G. SchönГEurophys. Letters 31Г31 (1995).

55. K. MullenГE. Ben-JacobГR. C. Jaklevic Гand Z. Schuss ГPhys. Rev. B 37Г98 (1988).

56. J. König ГН. SchoellerГand G. SchönГPhys. Rev. Lett. 78Г4482 (1997).

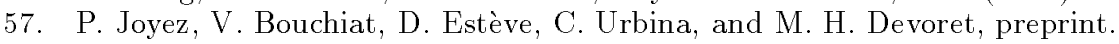

58. D. V. Averin and A. N. KorotkovГ Zh. Eksp. Teor. Fiz. 97Г 1661 (1990) [Sov. Phys. JETP 70Г937 (1990)]; D. V. AverinT A. N. KorotkovTand K. K. LikharevT Phys. Rev. B 44Г6199 (1991).

59. C. W. J. BeenakkerГPhys. Rev. B 44Г1646 (1991).

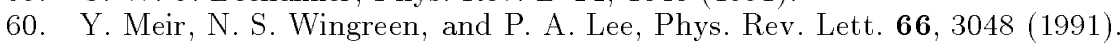

61. H. van HoutenГC. W. J. BeenakkerГand A. A. M. StaringГin Ref. [50].

62. D. WeinmannTW. Häusler $W$ W. PfaffГB. KramerTand U. WeissTEurophys. Lett. $26 \Gamma$ 467 (1994); D. Weinmann et al Phys. Rev. Lett. 74Г984 (1995).

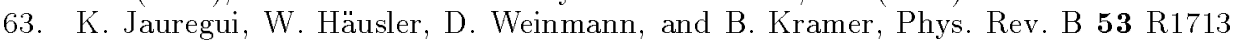
(1996).

64. D. Pfannkuche and S. E. UlloaГPhys. Rev. Lett. 74Г1194 (1995).

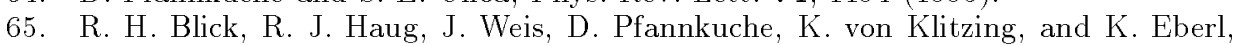


Phys. Rev. B 53Г7899 (1996).

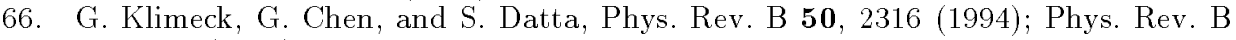
$\mathbf{5 0} \Gamma 8035$ (1994).

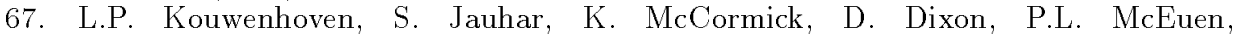
Yu.V. NazarovTN.C. van der VaartTand C.T. FoxonTPhys. Rev. B 50Г2019 (1994).

68. I. A. Devyatov and K. K. Likharev TPhysica B 194-196Г1341 (1994).

69. C. Bruder and H. SchoellerT Phys. Rev. Lett. 72Г1076 (1994).

70. G. Ingold and Yu. V. NazarovTin Ref. [50].

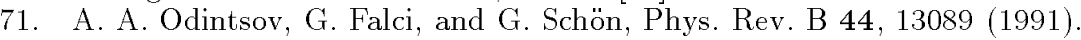

72. K. FlensbergTS. M. GirvinTM. JonsonTD. R. PennTand M. D. StilesГPhys. Scripta T 42Г189 (1992).

73. D. PfannkucheГV. GudmundssonTand P. A. MaksymГPhys. Rev. B 47Г2244 (1993).

74. W. HäuslerГAnn. Physik 5Г401 (1996). 